\title{
THE SDSS-IV EXTENDED BARYON OSCILLATION SPECTROSCOPIC SURVEY: QUASAR TARGET SELECTION
}

\author{
Adam D. Myers ${ }^{1}$, Nathalie Palangue-Delabrouille ${ }^{2}$, Abhishek Prakash $^{3}$, Isabelle Pâris ${ }^{4}$, Christophe Yeche ${ }^{2}$, \\ Kyle S. Dawson ${ }^{5}$, Jo Bovy ${ }^{6}$, Dustin Lang ${ }^{7,33}$, David J. Schlegel ${ }^{8}$, Jeffrey A. Newman ${ }^{3}$, Patrick Petitjean ${ }^{9}$, \\ Jean-Paul Kneib $^{10,11}$, Pierre Laurent ${ }^{2}$, Will J. Percival ${ }^{12}$, Ashley J. Ross ${ }^{12,13}$, Hee-Jong Seo ${ }^{14}$, Jeremy L. Tinker ${ }^{15}$, \\ Eric Armengaud ${ }^{2}$, Joel Brownstein ${ }^{5}$, Etienne Burtin ${ }^{2}$, Zheng Cai ${ }^{16}$, Johan Comparat ${ }^{17}$, Mansi Kasliwal ${ }^{18,34,35}$, \\ Shrinivas R. Kulkarni ${ }^{19}$, Russ Laher ${ }^{20}$, David Levitan ${ }^{21}$, Cameron K. McBride ${ }^{22}$, Ian D. McGreer ${ }^{16}$, \\ Adam A. Miller ${ }^{19,23,35}$, Peter Nugent ${ }^{8,24}$, Eran OfeK ${ }^{25}$, Graziano Rossi $^{26}$, John Ruan ${ }^{27}$, Donald P. Schneider ${ }^{28,29}$, \\ Branimir Sesar $^{30}$, Alina Streblyanska ${ }^{31,32}$, and Jason Surace ${ }^{20}$ \\ ${ }^{1}$ Department of Physics and Astronomy, University of Wyoming, Laramie, WY 82071, USA; amyers14@uwyo.edu \\ ${ }^{2}$ CEA, Centre de Saclay, Irfu/SPP, F-91191 Gif-sur-Yvette, France \\ ${ }^{3}$ Department of Physics and Astronomy and PITT PACC, University of Pittsburgh, Pittsburgh, PA 15260, USA \\ ${ }_{5}^{4}$ INAF-Osservatorio Astronomico di Trieste, Via G. B. Tiepolo 11, I-34131 Trieste, IT, Italy \\ ${ }^{5}$ Department of Physics and Astronomy, University of Utah, Salt Lake City, UT 84112, USA \\ ${ }^{6}$ Department of Astronomy and Astrophysics, University of Toronto, 50 St. George Street, Toronto, ON, M5S 3H4, Canada \\ ${ }^{7}$ Bruce and Astrid McWilliams Center for Cosmology, Department of Physics, Carnegie Mellon University, 5000 Forbes Ave, Pittsburgh, PA 15213, USA \\ ${ }^{8}$ Lawrence Berkeley National Laboratory, One Cyclotron Road, Berkeley, CA 94720, USA \\ ${ }^{9}$ UPMC-CNRS, UMR 7095, Institut d'Astrophysique de Paris, 98 bis Boulevard Arago, F-75014, Paris, France \\ ${ }^{10}$ Laboratoire d'Astrophysique, Ecole Polytechnique Fédérale de Lausanne Observatoire de Sauverny, 1290 Versoix, Switzerland \\ Aix Marseille Université, CNRS, LAM (Laboratoire d'Astrophysique de Marseille), UMR 7326, F-13388, Marseille, France \\ ${ }^{12}$ Institute of Cosmology \& Gravitation, Dennis Sciama Building, University of Portsmouth, Portsmouth, PO1 3FX, UK \\ ${ }_{13}$ Center for Cosmology and AstroParticle Physics, The Ohio State University, Columbus, OH 43210, USA \\ ${ }^{14}$ Department of Physics and Astronomy, Ohio University, 251B Clippinger Labs, Athens, OH 45701, USA
${ }^{15}$ Center for Cosmology and Particle Physics, Department of Physics, New York University, 4 Washington Place, New York, NY 10003, USA \\ ${ }^{16}$ Steward Observatory, University of Arizona, 933 N Cherry Ave, Tucson, AZ 85721, USA \\ ${ }^{17}$ Instituto de Física Teórica, (UAM/CSIC), Universidad Autónoma de Madrid, Cantoblanco, E-28049 Madrid, Spain \\ ${ }^{18}$ The Observatories, Carnegie Institution for Science, 813 Santa Barbara Street, Pasadena, CA 91101, USA \\ ${ }^{19}$ California Institute of Technology, Pasadena, CA 91125, USA \\ ${ }^{20}$ Spitzer Science Center, California Institute of Technology, M/S 314-6, Pasadena, CA 91125, USA \\ ${ }^{21}$ Division of Physics, Mathematics and Astronomy, California Institute of Technology, Pasadena, CA 91125, USA \\ ${ }^{22}$ Harvard-Smithsonian Center for Astrophysics, Harvard University, 60 Garden St., Cambridge MA 02138, USA \\ ${ }^{23}$ Jet Propulsion Laboratory, California Institute of Technology, 4800 Oak Grove Drive, MS 169-506, Pasadena, CA 91109, USA \\ ${ }^{24}$ Department of Astronomy, University of California, Berkeley, CA 94720, USA \\ ${ }^{25}$ Department of Particle Physics and Astrophysics, The Weizmann Institute of Science, Rehovot 76100, Israel \\ ${ }^{26}$ Department of Astronomy and Space Science, Sejong University, Seoul, 143-747, Korea \\ ${ }^{27}$ Department of Astronomy, University of Washington, Box 351580, Seattle, WA 98195, USA \\ ${ }^{28}$ Department of Astronomy and Astrophysics, 525 Davey Laboratory, The Pennsylvania State University, University Park, PA 16802, USA \\ ${ }^{29}$ Institute for Gravitation and the Cosmos, The Pennsylvania State University, University Park, PA 16802, USA \\ ${ }_{31}$ Max-Planck-Institut für Astronomie, Königstuhl 17, D-69117 Heidelberg, Germany \\ ${ }^{31}$ Instituto de Astrofisica de Canarias (IAC), E-38200 La Laguna, Tenerife, Spain \\ ${ }^{32}$ Universidad de La Laguna (ULL), Dept. Astrofisica, E-38206 La Laguna, Tenerife, Spain \\ Received 2015 August 19; accepted 2015 October 29; published 2015 December 2
}

\begin{abstract}
As part of the Sloan Digital Sky Survey (SDSS) IV the extended Baryon Oscillation Spectroscopic Survey (eBOSS) will improve measurements of the cosmological distance scale by applying the Baryon Acoustic Oscillation (BAO) method to quasar samples. eBOSS will adopt two approaches to target quasars over $7500 \mathrm{deg}^{2}$. First, a "CORE" quasar sample will combine the optical selection in ugriz using a likelihood-based routine called $X D Q S O z$, with a mid-IR-optical color cut. eBOSS CORE selection (to $g<22$ or $r<22$ ) should return $\sim 70 \mathrm{deg}^{-2}$ quasars at redshifts $0.9<z<2.2$ and $\sim 7 \mathrm{deg}^{-2} z>2.1$ quasars. Second, a selection based on variability in multi-epoch imaging from the Palomar Transient Factory should recover an additional $\sim 3-4 \mathrm{deg}^{-2} z>2.1$ quasars to $g<22.5$. A linear model of how imaging systematics affect target density recovers the angular distribution of $e$ BOSS CORE quasars over $96.7 \%$ (76.7\%) of the SDSS north (south) Galactic Cap area. The $e B O S S$ CORE quasar sample should thus be sufficiently dense and homogeneous over $0.9<z<2.2$ to yield the first few-percent-level BAO constraint near $\bar{z} \sim 1.5$.eBOSS quasars at $z>2.1$ will be used to improve BAO measurements in the Ly $\alpha$ Forest. Beyond its key cosmological goals, eBOSS should be the next-generation quasar survey, comprising $>500,000$ new quasars and $>500,000$ uniformly selected spectroscopically confirmed $0.9<z<2.2$ quasars. At the conclusion of $e B O S S$, the SDSS will have provided unique spectra for more than 800,000 quasars.
\end{abstract}

Key words: catalogs - cosmology: observations - galaxies: distances and redshifts - galaxies: photometry methods: data analysis - quasars: general

\footnotetext{
33 McWilliams fellow.

34 Carnegie-Princeton Fellow.

${ }^{35}$ Hubble fellow.
} 


\section{INTRODUCTION}

More than 50 years have elapsed since it was discovered that quasars are bright, blue, extragalactic sources in optical imaging (Schmidt 1963) and that the vast majority of unresolved, extragalactic objects that are bluer than the stellar main sequence are quasars (Sandage 1965). Since then, many imaging surveys have used a UV-excess (UVX) criterion, as manifested in simple optical color cuts, to provide a mechanism for targeting quasars (e.g., Sandage \& Luyten 1969; Braccesi et al. 1970; Formiggini et al. 1980; Green et al. 1986; Boyle et al. 1990). The UVX approach, which mainly targets quasars at redshifts around $0.5<z<2.5$, precipitated increasingly extensive spectroscopically confirmed quasar samples as the capabilities of imaging surveys improved, such as the Large Bright Quasar Survey (Hewett et al. 1995), the 2dF QSO Redshift Survey (Croom et al. 2004), and the 2dF-SDSS LRG and QSO Survey (Croom et al. 2009).

Modifications of the UVX approach to target all of color space beyond the stellar locus, rather than just the blue side (e.g., Warren et al. 1987; Kennefick et al. 1995; Newberg \& Yanny 1997), extended the selection of large numbers of quasars to $z>2.5$. The Sloan Digital Sky Survey (SDSS; York et al. 2000) applied this methodology to imaging taken using a new ugriz filter system (Fukugita et al. 1996). SDSS eventually spectroscopically confirmed an unprecedentedly large sample of more than 100,000 quasars (Richards et al. 2002; Schneider et al. 2010) as part of the SDSS-I and II surveys.

In addition to optical color space, SDSS-I and II selected about $10 \%$ of their quasar samples via radio matches to the FIRST survey (Becker et al. 1995; Helfand et al. 2015), or $\mathrm{X}$-ray matches to the ROSAT All Sky Survey (Voges et al. 1999). The proliferation of such large multi-wavelength surveys, as well as multi-epoch surveys, has made quasar classification approaches that do not rely on optical colors (but still may use optical imaging to constrain morphology or brightness) increasingly attractive. Such approaches include the use of the radio (e.g., White et al. 2000; McGreer et al. 2009), near-infrared (e.g., Banerji et al. 2012), or both (e.g., Glikman et al. 2012); the lack of an observed proper motion (e.g., Kron $\&$ Chiu 1981), the use of the mid-infrared (e.g., Lacy et al. 2004; Stern et al. 2005; Richards et al. 2009a; Stern et al. 2012), X-rays (e.g., Trichas et al. 2012), or both (e.g., Lacy et al. 2007; Hickox et al. 2007, 2009); the use of slitless spectroscopy (e.g., Osmer 1982; Schmidt et al. 1986); and the use of variability (e.g., Usher 1978; Rengstorf et al. 2004a; Schmidt et al. 2010; Butler \& Bloom 2011; MacLeod et al. 2011; Palanque-Delabrouille et al. 2011).

Even after the first iterations of the SDSS, the selection of quasars at $z \gtrsim 2.5$ remained relatively incomplete. This problem arose partially because SDSS-I and II targeted quasars that were a magnitude or more brighter than the limits of SDSS imaging, thus sampling only the high luminosity regime at these redshifts, and partially because the stellar and quasar loci intersect in ugriz color space around the "quasar redshift desert" near $z \sim 2.7$ (Fan 1999). In order to target quasars at $z>2.1$ for cosmological studies of the Ly $\alpha$ Forest, the SDSSIII (Eisenstein et al. 2011) Baryon Oscillation Spectroscopic Survey (BOSS; Dawson et al. 2013) attempted to circumvent these problems of quasar selection near $z \sim 3$ by applying sophisticated, multi-wavelength, multi-epoch star-quasar separation techniques to the full depth of SDSS imaging. BOSS spectroscopically identified $\sim 170,000$ new quasars of redshift
$2.1 \leqslant z<3.5$ to a depth of $g<22$ (I. Pâris et al. 2016, in preparation; henceforth $D R 12 Q$ ), a sample about 10 times larger than for the same redshift range in SDSS-I and II. BOSS may only be $\sim 60 \%$ complete (e.g., Ross et al. 2013), raising the possibility that there are additional $g<22$ quasars to be discovered in this redshift regime.

In combination, SDSS-I/II/III targeted quasars at $2.1 \lesssim z \lesssim 4$ to a magnitude limit of $g<22$ or $r<21.85$ (Ross et al. 2012), and quasars at all redshifts to $i<19.1^{36}$ (Richards et al. 2002). There remains an obvious, highly populated discovery space using SDSS imaging data-namely, $z<2.1$ quasars fainter than $i=19.1$. In addition, since the advent of BOSS, new and extensive multi-wavelength and multi-epoch imaging has become available, allowing $z>2.1$ quasars to be targeted that may have been missed by BOSS. In particular, mid-IR colors provide a powerful mechanism for separating quasars and stars, which means that Wide-field Infrared Survey Explorer (WISE; Wright et al. 2010) data provide additional information for targeting quasars that otherwise resemble stars in optical color space (e.g., Stern et al. 2012; Assef et al. 2013; Yan et al. 2013).

The remaining potential of SDSS and other imaging for targeting new quasars has obvious synergy with the now mature field of using Baryon Acoustic Oscillation features (BAOs) to measure the expansion of the universe (Eisenstein et al. 1998; Linder 2003; Seo \& Eisenstein 2003). No strong BAO constraint currently exists in the redshift range $1 \lesssim z \lesssim 2$, and $\mathrm{BAO}$ measurements at yet higher redshift remain a particularly potent constraint on the evolution of the angular diameter distance, $D_{A}(z)$, and of the Hubble Parameter, $H(z)$ (Aubourg et al. 2014). These factors led to the conception of a new survey-the extended Baryon Oscillation Spectroscopic Survey (eBOSS; Dawson et al. 2015) as part of SDSS-IV.

It has been difficult to detect BAO features using quasars as direct tracers due to their low space density. eBOSS will circumvent this issue by surveying quasars over a huge volume, corresponding to $7500 \mathrm{deg}^{2}$ of sky. The quasar component of $e B O S S$ will attempt to statistically target and measure redshifts for $\sim 500,000$ quasars at $0.9<z<2.2$ (including spectroscopically confirmed quasars from SDSS-I/ II, which will not need to be retargeted). We refer to this homogeneous tracer sample as the $e B O S S$ CORE quasar target selection. BOSS targeted quasars at $z>2.2$ with the main goal of using them as indirect tracers to study cosmology in the Ly $\alpha$ Forest. In contrast, $e B O S S$ will open up the $i>19.1, z<2.2$ parameter space to use the quasars as cosmological tracers.

In addition, analyses of the Ly $\alpha$ Forest with BOSS have provided substantial new insights into cosmological constraints (e.g., Slosar et al. 2011, 2013; Noterdaeme et al. 2012; Busca et al. 2013; Kirkby et al. 2013; Palanque-Delabrouille et al. 2013b; Font-Ribera et al. 2014; Delubac et al. 2015). $e B O S S$ will also (heterogeneously) observe more than $\sim 60,000$ new $z>2.1$ quasars and will reobserve low signal-to-noise ratio $(\mathrm{S} / \mathrm{N}) z>2.1$ quasars from $B O S S$. The main goals of this targeting campaign are to produce measurements of the BAO scale (in both $d_{A}(z)$ and $\left.H(z)\right)$ in the Ly $\alpha$ Forest that approach $\sim 1.5 \%$ at $z \sim 2.5$ and that probe an entirely new redshift regime via quasar clustering at $z \sim 1.5$ with $\sim 2 \%$ precision (see Section 2).

\footnotetext{
${ }^{36}$ In addition, smaller dedicated programs affiliated with SDSS have targeted higher-redshift quasars to fainter limits.
} 
In total, at the conclusion of $e B O S S$, the SDSS surveys will have spectroscopically confirmed more than 800,000 quasars. The scope of the science that can be conducted with a large sample of quasars across a range of redshifts has been shown to be vast. Beyond Ly $\alpha$ Forest science, BOSS also facilitated additional, diverse quasar science, from measurements of quasar clustering and the quasar luminosity function to studies of Broad Absorption Line quasars (e.g., Filiz et al. 2012, 2013, 2014; White et al. 2012; Alexandroff et al. 2013; Finley et al. 2013; McGreer et al. 2013; Ross et al. 2013; Vikas et al. 2013; Greene et al. 2014; Eftekharzadeh et al. 2015). eBOSS will seek to augment many of these measurements. In addition to higherredshift studies, SDSS-IV/eBOSS will produce a $z<2.2$ sample of quasars about six times larger than the final SDSSII quasar catalog (Schneider et al. 2010) and will further benefit from upgrades conducted for SDSS-III (such as larger wavelength coverage for spectra; see Smee et al. 2013, for extensive details of upgrades). Many high-impact projects that used the original SDSS-I/II quasar samples can therefore potentially be revisited using much larger samples with $e B O S S$, such as composite quasar spectra, rare types of quasars, and precision studies of the quasar luminosity function (e.g., Vanden Berk et al. 2001; Inada et al. 2003; McLure \& Dunlop 2004; Hennawi et al. 2006; Richards et al. 2006; York et al. 2006; Kaspi et al. 2007; Netzer \& Trakhtenbrot 2007; Shen et al. 2008; Boroson \& Lauer 2009).

In this paper, we describe quasar target selection for the SDSS-IV/eBOSS survey. Further technical details about $e B O S S$ can be found in our companion papers, which include an overview of $e B O S S$ (Dawson et al. 2015) and discussions of targeting for Luminous Red Galaxies (Prakash et al. 2015b; see also Prakash et al. 2015a), and Emission Line Galaxies (Comparat et al. 2015). eBOSS will run concurrently with two surveys; the SPectroscopic IDentification of ERosita Sources survey (SPIDERS) and the Time Domain Spectroscopic Survey (TDSS; Morganson et al. 2015). These associated surveys are further outlined in our companion overview paper (Dawson et al. 2015).

In Section 2 we discuss how forecasts for BAO constraints at different redshifts drive targeting goals for $e B O S S$ quasars. The parent imaging used for eBOSS quasar target selection is outlined in Section 3. Those interested in the main quasar targeting details for eBOSS (e.g., targeting algorithms, the meaning of targeting bits, the criteria for retargeting of previously known quasars) should see Section 4 of this paper. In Section 5, we use the results from an extensive pilot survey (SEQUELS; The Sloan Extended QUasar, ELG and LRG Survey, undertaken as part of SDSS-III) to detail our expected efficiency and distribution of quasars for eBOSS. An important criterion for any large-scale structure survey is sufficient homogeneity to facilitate modeling of the distribution of the tracer population-the "mask" of the survey. In Section 6 we use the full eBOSS target sample to characterize the homogeneity of $e B O S S$ quasar selection. In Section 7, we provide our overall conclusions regarding $e B O S S$ quasar targeting, as well as a bulleted summary of the final $e B O S S$ CORE quasar selection algorithm.

Unless we state otherwise, all magnitudes and fluxes in this paper are corrected for Galactic extinction using the dust maps of Schlegel et al. (1998). Specifically, we use the correction based upon the recalibration of the SDSS reddening coefficients measured by Schlafly \& Finkbeiner (2011). For WISE we adopt the reddening coefficients from Fitzpatrick (1999). The SDSS photometry has been demonstrated to have colors that are within 3\% (Schlafly \& Finkbeiner 2011) of being on the AB system (Oke \& Gunn 1983). WISE is calibrated to be on the Vega system. We use a cosmology of $\left(\Omega_{\mathrm{m}}, \Omega_{\Lambda}, h \equiv H_{0} /\right.$ $\left.100 \mathrm{~km} \mathrm{~s}^{-1} \mathrm{Mpc}^{-1}\right)=(0.315,0.685,0.67)$ that is consistent with recent results from Planck (Planck Collaboration et al. 2014).

\section{COSMOLOGICAL GOALS OF $e B O S S$ AND IMPLICATIONS FOR QUASAR TARGET SELECTION}

\subsection{CORE and Ly $\alpha$ Quasars}

The goal of the $e B O S S$ quasar survey is to study the scale of the BAO in two distinct redshift regimes: $z \sim 1.5$ using the clustering of quasars, and $z \sim 2.5$ using high-redshift quasars as backlights to illuminate the $\operatorname{Ly} \alpha$ Forest. Broadly, this approach requires a sample of statistically selected quasars in the redshift range $0.9<z<2.2$ (which we will refer to as "CORE quasars") and quasars selected at $z>2.1$ (which we will refer to as "Ly $\alpha$ quasars").

A major difference between the two samples is the homogeneity of the target selection technique. The selection of CORE quasars must be statistically uniform. Ly $\alpha$ quasars, however, can be selected heterogeneously, because a clustering measurement using the Ly $\alpha$ Forest does not require the background quasars to have a uniform (or even a reproducible) selection. In fact, the full redshift range of the CORE sample will extend well beyond $0.9<z<2.2$, and many CORE quasars can thus be utilized as Ly $\alpha$ quasars. The terminology "CORE quasars" therefore refers to how the quasars were targeted, whereas the terminology "Ly $\alpha$ quasars" refers to the redshift of the quasar.

\subsection{Target Requirements for CORE and Lya Quasars}

Full details of the techniques used to forecast requirements for the eBOSS quasars are provided in our companion overview paper (Dawson et al. 2015). Those forecasts imply the following broad requirements for quasar target selection, driven by instrument capabilities and a $2 \%$ measurement of the BAO distance scale (G. Zhao et al. 2016, in preparation). For the CORE quasars:

1. survey area $>7500 \mathrm{deg}^{2}$;

2. total number of $0.9<z<2.2$ quasars $>435,000$ (this corresponds to $58 \mathrm{deg}^{-2}$ over exactly $7500 \mathrm{deg}^{2}$ );

3. a total density of assigned fibers of $<90 \mathrm{deg}^{-2}$ (effectively a target density of $\lesssim 115 \mathrm{deg}^{-2}$ for reasons noted at the end of this section);

4. redshift precision ${ }^{37}<300 \mathrm{~km} \mathrm{~s}^{-1}$ rms for $z<1.5$ and $(300+400(z-1.5)) \mathrm{km} \mathrm{s}^{-1}$ for $z>1.5$;

5. catastrophic redshift errors (exceeding $3000 \mathrm{~km} \mathrm{~s}^{-1}$ ) $<1 \%$, where the redshifts are not known to be in error;

6. maximum absolute variation in expected target density as a function of imaging survey sensitivity, stellar density, and Galactic extinction of $<15 \%$ within the survey footprint;

\footnotetext{
37 see the $e B O S S$ overview paper (Dawson et al. 2015) for a discussion of this requirement and Hewett \& Wild (2010) for details of the precision of SDSS quasar redshifts.
} 
7. maximum fluctuations in target density due to imaging zero-point errors of $<15 \%$ in each individual band used for targeting.

Once these CORE requirements are met, the remaining fibers not allocated to other $e B O S S$ target classes are assigned to the Ly $\alpha$ target class. These Ly $\alpha$ quasars have the following additional constraints and requirements.

1. BOSS quasars within the eBOSS area with $\mathrm{S} / \mathrm{N}^{38}$ pixel $^{-1}=0$, or $0.75<\mathrm{S} / \mathrm{N} \mathrm{pixel}^{-1}<3$ must be reobserved.

2. Flux calibration at least as accurate as BOSS.

3. Recalibration of the BOSS high- $z$ quasar sample using a spectroscopic pipeline that is consistent with that of eBOSS.

A subtlety arises for item (3) of the CORE requirements; targets with existing good spectroscopy from earlier iterations of the SDSS are not assigned fibers as part of $e B O S S$ (see Section 4.4.10). On average, this saves 25 fibers $\mathrm{deg}^{-2}$. Therefore, this paper will typically quote a total target density of $115 \mathrm{deg}^{-2}$, but this corresponds to a density of assigned fibers of only $90 \mathrm{deg}^{-2}$ for CORE quasars.

\section{PARENT IMAGING FOR TARGET SELECTION}

\subsection{Updated Calibrations of SDSS Imaging}

All eBOSS quasar targets are ultimately tied to the SDSS-I/ II/III images collected in the ugriz system (Fukugita et al. 1996) using the wide-field imager (Gunn et al. 1998) on the SDSS telescope (Gunn et al. 2006). SDSS-I/II mostly derived imaging over the $\sim 8400 \mathrm{deg}^{2}$ "Legacy" area, $90 \%$ of which was in the north Galactic Cap (NGC). This imaging was released as part of SDSS Data Release 7 (DR7; Abazajian et al. 2009). The legacy imaging area of the SDSS was expanded by $\sim 2500 \mathrm{deg}^{2}$ in the south Galactic Cap (SGC) as part of DR8 (Aihara et al. 2011). The SDSS-III/BOSS survey used DR8 imaging for target selection over $\sim 7600 \mathrm{deg}^{2}$ in the NGC and $3200 \mathrm{deg}^{2}$ in the SGC (Dawson et al. 2013). Quasar targets are being selected for $e B O S S$ over the same areas as $B O S S$, and ultimately $e B O S S$ will observe quasars over a subset of at least $7500 \mathrm{deg}^{2}$ of this area.

Although adopting the same area as BOSS, eBOSS target selection takes advantage of the updated calibrations of the SDSS imaging. Schlafly et al. (2012) applied the "ubercalibration" technique of Padmanabhan et al. (2008) to PanSTARRS imaging (Kaiser et al. 2010), achieving an improved global calibration compared with SDSSDR8. Targeting for eBOSS is conducted using SDSS imaging that is calibrated to the Schlafly et al. (2012) Pan-STARRS solution, as fully detailed in D. Finkbeiner et al. (2016, in preparation). We will refer to this set of observations as the "updated" imaging.

The specific version of the updated SDSS imaging used in $e B O S S$ target selection is stored in the calib_obj or "data sweep" files (Blanton et al. 2005). These data correspond to the

\footnotetext{
${ }^{38} \mathrm{~S} / \mathrm{N}$ is defined as the mean $\mathrm{S} / \mathrm{N}$ per Ly $\alpha$ Forest pixel measured over the restframe wavelength range of $1040 \AA<\lambda<1200 \AA$. A "pixel" here refers to a single bin of wavelength in a BOSS spectrum. The logic behind retargeting the $\mathrm{S} / \mathrm{N}$ pixel $^{-1}=0$ spectra is that they are almost certainly bad, whereas $0 \leqslant \mathrm{~S} / \mathrm{N} \mathrm{pixel}^{-1}<0.75$ spectra are good, but of irrecoverably low $\mathrm{S} / \mathrm{N}$ (see Section 4.2.2).

39 e.g., http://data.sdss3.org/datamodel/files/PHOTO_SWEEP/RERUN/ calibObj.html
}

native files used in the SDSS-III data model ${ }^{39}$ and the updated Pan-STARRS-calibrated data sweeps will be made available in a future SDSS Data Release. The magnitudes derived from these data sweeps are AB magnitudes (not, e.g., asinh "Luptitudes"; Lupton et al. 1999). Note that the XDQSOz targeting technique (Bovy et al. 2012) adopted by eBOSS is designed to handle noisy data, so it can rigorously incorporate small (and even negative) fluxes when classifying quasars.

\subsection{WISE}

The WISE (Wright et al. 2010) surveyed the full sky in four mid-infrared bands centered on 3.4, 4.6, 12, and $22 \mu \mathrm{m}$, known as $\mathrm{W} 1, \mathrm{~W} 2, \mathrm{~W} 3$, and $\mathrm{W} 4$. For $e B O S S$ we only use the $\mathrm{W} 1$ and W2 bands, which are substantially deeper than W3 and W4. Over the course of its primary mission and "NEOWISE postcryo" continuation, WISE completed two full scans of the sky in W1 and W2. More than $99 \%$ of the sky has 23 or more exposures in $\mathrm{W} 1$ and $\mathrm{W} 2$; the median coverage is 33 exposures. We investigate whether the non-uniform spatial distribution of WISE exposure depth presents a problem for modeling CORE quasar clustering in Section 6.

We use the "unWISE" coadded photometry from Lang (2014) applied to SDSS imaging sources (as detailed in Lang et al. 2014). This approach produces forced photometry of custom coadds of the WISE imaging at the positions of all $S D S S$ primary sources. Using forced photometry rather than catalog-matching avoids issues such as blended sources and non-detections. Because the WISE scale is $2.175 \mathrm{pixel}^{-1}$ (roughly seven times as large as $S D S S$ ), and many of our targets have WISE fluxes below the "official" WISE catalog detection limits, using forced photometry is of significant benefit.

\subsection{Palomar Transient Factory (PTF)}

The $P T F^{40}$ is a wide-field photometric survey aimed at a systematic exploration of the optical transient sky via repeated imaging over 20,000 deg in the northern Hemisphere (Law et al. 2009; Rau et al. 2009). The PTF image processing is presented in Laher et al. (2014), while the photometric calibration, system, and filters are discussed in Ofek et al. (2012). In 2013 February, the next phase of the program, $i P T F$ (intermediate $P T F$ ) began. Both surveys use the CFHT12K mosaic camera, mounted on the $1.2 \mathrm{~m}$ Samuel Oschin Telescope at Palomar Observatory. The camera has an $8.1 \mathrm{deg}^{2}$ field of view and $1^{\prime \prime}$ sampling. Because one detector (CCD03) is non-functional, the usable field of view is reduced to $7.26 \mathrm{deg}^{2}$. Observations are mostly performed in the Mould$R$ broadband filter, with some in the SDSS $g$-filter. Under median seeing conditions, the images are obtained with 2 !" 0 FWHM, and reach $5 \sigma$ limiting $\mathrm{AB}$ magnitudes of $m_{R} \simeq 20.6$ and $m_{g^{\prime}} \simeq 21.3$ in $60 \mathrm{~s}$ exposures. The cadence varies between fields, and can produce one measurement every five nights in regions of the sky dedicated to supernova searches. Four years of PTF survey operations have yielded a coverage of $\sim 90 \%$ of the $e B O S S$ footprint.

Two automated data processing pipelines are used in parallel in the search for transients: a near-real-time image subtraction pipeline at Lawrence Berkeley National Laboratory, and a database populated on timescales of a few days at the Infrared

\footnotetext{
${ }^{40}$ See http://irsa.ipac.caltech.edu/Missions/ptf.html for the public PTF data.
} 
Processing and Analysis Center (IPAC). The eBOSS analysis uses the individual calibrated frames available from IPAC (Laher et al. 2014).

We developed a customized pipeline based on the SWarp (Bertin et al. 2002) and SCAMP (Bertin 2006) public packages to build coadded PTF images on a timescale adapted to quasar targeting (i.e., typically 1-4 epochs per year, depending on the cadence and total exposure time within each field). Using the same algorithms, a full stack is also constructed by coadding all available images. This full stack is complete at $3 \sigma$ to $g \sim 22.0$, and has more than $50 \%$ completeness to quasars at $g \sim 22.5$. The full stack is used to extract a catalog of PTF sources from each of the coadded PTF images. The light curves (flux as a function of time) for all of these PTF sources are measured.

\section{QUASAR TARGET CLASSES}

As only a limited number of fibers are available in the eBOSS experiment, each target class is assigned a different target density to optimize the scientific return. eBOSS will attempt to make the first $2 \%$ measurement of the BAO scale at a redshift near $z \sim 1.5$, and the uniqueness of this measurement led to statistically selected $0.9<z<2.2$ quasars being prioritized at a density of $90 \mathrm{deg}^{-2}$ fibers. As noted in Section 2.2, because objects targeted by past SDSS projects do not need to be reobserved, this fiber allocation effectively corresponds to a density of $115 \mathrm{deg}^{-2}$ targets. eBOSS will also attempt to augment BOSS measurements of clustering in the Ly $\alpha$ Forest, improving BAO constraints from near $2 \%$ to closer to $1.5 \%$. This program is assigned the remaining available eBOSS fibers once other target classes have been accounted for, typically resulting in $\sim 20 \mathrm{deg}^{-2}$ targets. The combined cosmological constraints that can be achieved by this overall program design are detailed in G. Zhao et al. (2016, in preparation).

As further discussed in Section 2, this creates two distinct target classes in $e B O S S$ : CORE quasars and Ly $\alpha$ quasars. The CORE quasars are targeted in a statistically reproducible fashion, with the intention of using them to measure clustering over redshifts of $0.9<z<2.2$. The $\operatorname{Ly} \alpha$ quasars are targeted to lie at $z>2.1$ to augment the BAO signal detected by BOSS. These two categories of quasars are not mutually exclusive, in that the CORE quasars are not constrained to lie at $z<2.1$ and so the CORE selection algorithm can also identify Ly $\alpha$ quasars. In the rest of this section we discuss each $e B O S S$ target class in detail. The full targeting algorithm is also depicted by a flow-chart in Figure 1.

\subsection{Broad Overview of the CORE Quasar Sample}

The eBOSS CORE sample is designed to provide a statistically selected sample of $115 \mathrm{deg}^{-2}$ targets that-after eBOSS spectroscopy of the $90 \mathrm{deg}^{-2}$ targets that do not have existing good SDSS spectra-comprises $>58 \mathrm{deg}^{-2}$ total quasars with accurate redshifts in the range $0.9<z<2.2$ (see Section 2). This $>58 \mathrm{deg}^{-2}$ quasars will consist of both new quasars from $e B O S S$ spectroscopy and previously known quasars from the sample of $25 \mathrm{deg}^{-2}$ targets that have existing SDSS spectroscopy. To achieve this goal eBOSS uses two complementary methods: an optical selection using the $X D Q S O z$ method of Bovy et al. (2012), and a mid-IR-optical color cut using WISE imaging. The specifics of these two methods are detailed in the next few sections.
The starting sample for CORE targeting is all point sources in SDSS imaging that are PRIMARY, have (de-extincted) PSF magnitudes of $g<22$ or $r<22$ and a FIBER2MAG ${ }^{41}$ of $i>17$, and have good IMAGE_STATUS. ${ }^{42}$ These basic initial cuts are discussed further in Section 4.3.

Point sources in the SDSS are denoted by the flag obj c_type $==6$, corresponding to a magnitude cut based on star-like or galaxy-like profile fits of psfMag - modelMag $\leqslant 0.145$ (Stoughton et al. 2002). A concern might be that a selection to $r \sim 22$ might suffer incompleteness to quasars at $r \gtrsim 21$, where star-galaxy separation in SDSS imaging was initially argued to break down due to errors on profile fits (e.g., Stoughton et al. 2002; Scranton et al. 2002). In general, however, at the limit of the SDSS imaging the trend is to classify faint, ambiguous sources as point-like. The expectation is then that a selection approaching $r \sim 22$ will become increasingly contaminated by galaxies that are classified as unresolved, rather than miss quasars that are classified as resolved (see also the discussion in Section 4.5.1 of Richards et al. 2009b). Further, requiring objc_type $==6$ and applying $\mathrm{XDQSOz}$ reduces galaxy contamination to $\lesssim 10 \%$, even at $i \sim 22$ (see Figure 11 of Bovy et al. 2012), so we expect our selection to remain robust even to $r \sim 22$ (which, on average, corresponds to $i \sim 21.85$ for $0.9<z<2.2$ quasars).

From the initial sample of magnitude-limited PRIMARY point sources, objects are targeted if they have an $\mathrm{XDQSOz}$ probability of being a quasar at $z>0.9$ of more than $20 \%$ (i.e., $\operatorname{PQSO}(z>0.9)>0.2)$. It is important to note the subtle distinction between the specific goal of the CORE sample and the sample it produces. The goal of CORE is to uniformly target $>58 \mathrm{deg}^{-2}$ quasars in the redshift range $0.9<z<2.2$, but no attempt is made to restrict the upper redshift range of the CORE quasar sample. The CORE is left free to recover quasars at $z>2.2$ because, although such quasars are outside the preferred CORE redshift range, they remain useful as tracers of the Ly $\alpha$ Forest. To this moderate-probability $X D Q S O z$ sample, a WISE-optical color cut is applied to further reduce the target density by filtering out obvious stars based on optical-mid-IR colors. Finally, objects are not targeted if they have existing good spectroscopy from earlier iterations of the SDSS unless a visual inspection as part of BOSS produced an ambiguous classification. The resulting set of objects comprises the $e B O S S$ CORE quasar sample.

\subsection{1. $\mathrm{XDQSOz}$}

$X D Q S O$ (Bovy et al. 2011a) is a method of classifying quasars in flux-space using extreme deconvolution (XD; Bovy et al. 2011b) to estimate the density distribution of quasars as compared to non-quasars. Effectively, XDQSO takes any test point in flux-space, together with its flux errors, and convolves that error envelope with deconvolved distributions of the quasar and of the non-quasar loci. By weighting this convolution with a prior representing the expected numbers of quasars and non-quasars, the test point is assigned a probability of being a quasar. $X D Q S O$ inherits many desiderata from $X D$, including the rigorous incorporation of (and extrapolation from) errors on fluxes, and the ability to

\footnotetext{
41 FIBER2MAG corresponds to the flux through a fiber with a $2^{\prime \prime}$ diameter, appropriate to BOSS. Surveys with the SDSS spectrographs instead used FIBERMAG, appropriate to a $3^{\prime \prime}$ fiber diameter.

42 All target classes detailed in this paper undergo these cuts with the exception of the variability-selected sample discussed in Section 4.2.1.
} 


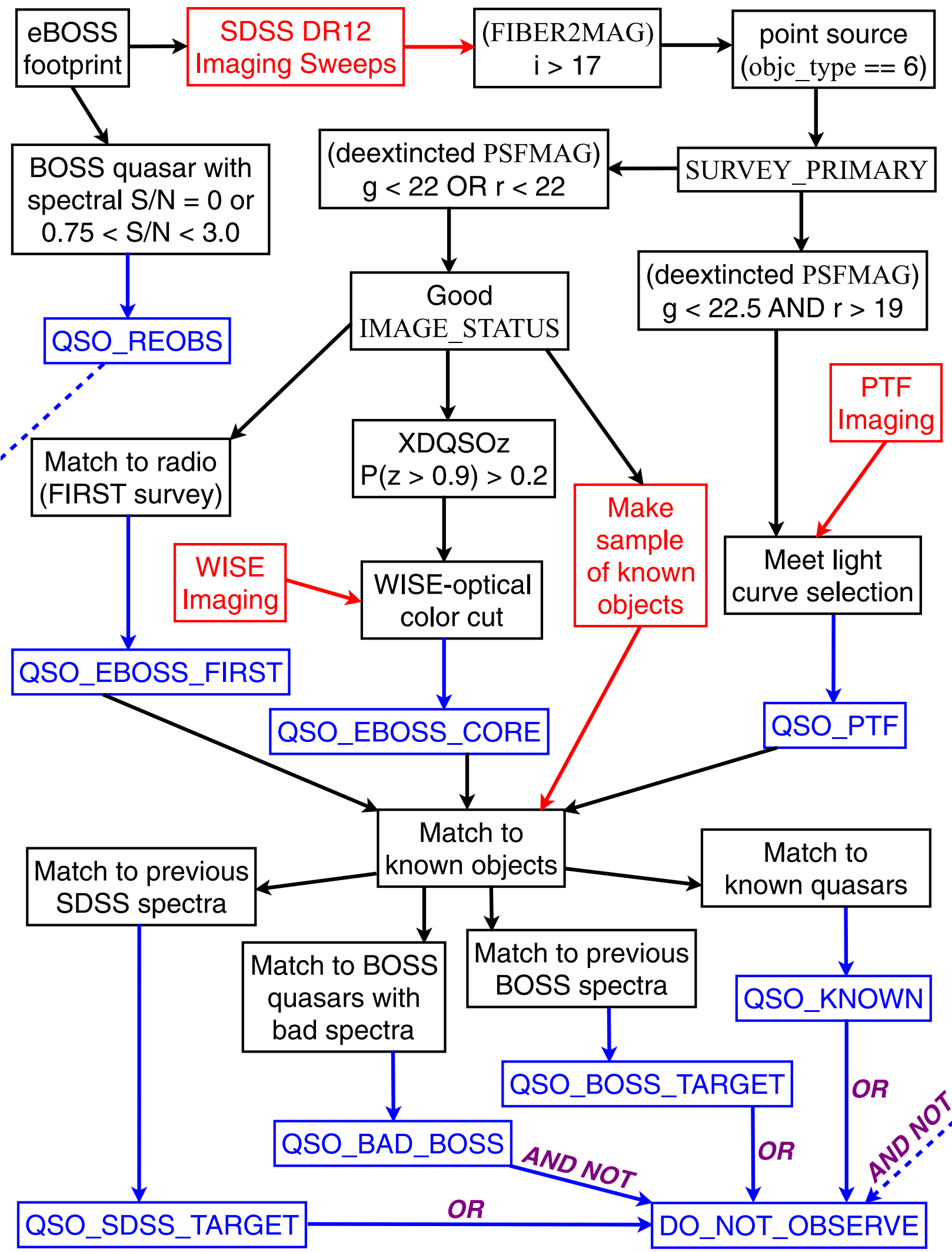

Figure 1. Flowchart depicting $e B O S S$ quasar target selection. Red boxes represent the sources of input information, such as imaging (see Section 3) or catalogs of known objects. Black boxes depict cuts that are made to the input sources as part of the target selection algorithm (see Section 4). Blue boxes depict output target selection bits (see Section 4.4). The Boolean terms in purple describe how the four bits produced by matching to previous spectra are combined to set the DO_NOT_OBSERVE bit (see Section 4.4.10). The dashed blue arrow indicates that QSO_REOBS targets are always reobserved, regardless of the value of DO_NOT_OBSERVE. The sample of known objects undergoes the CORE flag and magnitude cuts rather than the PTF magnitude cuts. Consequently, PTF selection could re-target previously known objects with bad IMAGE_STATUS and/or with $22<g<22.5$. 
Table 1

Efficiency of Quasar Target Selection in the CFHTLS-W3 Test Survey as a Function of $X D Q S O z$ Probability Cut

\begin{tabular}{|c|c|c|c|c|c|c|}
\hline \multirow{3}{*}{$\begin{array}{l}\text { ID } \\
\text { (Rows 1-4) } \\
z_{\text {spec range }} \\
\text { For quasars } \\
\text { (Rows 5-7) }\end{array}$} & \multicolumn{6}{|c|}{ PQSO } \\
\hline & \multicolumn{2}{|c|}{$\begin{array}{c}(z>0.0) \\
>0.2\end{array}$} & \multicolumn{2}{|c|}{$\begin{array}{c}(z>0.9) \\
>0.2\end{array}$} & \multicolumn{2}{|c|}{$\begin{array}{c}(z>0.0)>0.2 \\
\text { and } \\
(z>0.9)<0.2\end{array}$} \\
\hline & $N$ & $\%$ & $N$ & $\%$ & $N$ & $\%$ \\
\hline Stars & 27.0 & $18.2 \%$ & 23.3 & $16.8 \%$ & 3.6 & $39.6 \%$ \\
\hline Galaxies & 13.9 & $9.4 \%$ & 12.3 & $8.8 \%$ & 1.6 & $17.8 \%$ \\
\hline Unidentified & 2.4 & $1.6 \%$ & 2.2 & $1.6 \%$ & 0.2 & $2.0 \%$ \\
\hline Quasars & 105.0 & $70.8 \%$ & 101.3 & $72.8 \%$ & 3.7 & $40.6 \%$ \\
\hline$z<0.9$ & 13.2 & $8.9 \%$ & 10.9 & $7.9 \%$ & 2.3 & $24.8 \%$ \\
\hline $0.9<z<2.2$ & 70.9 & $47.8 \%$ & 69.7 & $50.1 \%$ & 1.2 & $12.9 \%$ \\
\hline$z>2.2$ & 20.9 & $14.1 \%$ & 20.7 & $14.9 \%$ & 0.3 & $3.0 \%$ \\
\hline Total & 148.3 & $100 \%$ & 139.1 & $100 \%$ & 9.2 & $100 \%$ \\
\hline
\end{tabular}

Note. The total survey area was $11.0 \mathrm{deg}^{2}$ and $N$, the number of spectroscopically confirmed targets, is always expressed in $\mathrm{deg}^{-2}$ over this area.

distinguish the effect on quasar probabilities of data that are completely missing from data that are merely of low significance. This feature is a boon for quasar classification near the limits of imaging data where flux errors are large. For eBOSS targeting, we adopt the $X D Q S O z$ method (Bovy et al. 2012), which extends the $X D Q S O$ schema to provide probabilistic classifications for quasars in any specified range of redshift.

In pursuit of the eBOSS CORE goal of $>58 \mathrm{deg}^{-2}$ $0.9<z<2.2$ quasars, a test spectroscopic survey in the W3 field of the CFHT Legacy Survey was conducted. ${ }^{43}$ This CFHTLS-W3 test survey was deemed necessary because no iteration of the SDSS-I/II/III specifically targeted quasars as faint as $r \sim 22$ over the redshift range $0.9<z<2.2$. Although the CFHTLS-W3 test survey informed the initial quasar target selection for $e B O S S$, and so will be used to describe the broad ideas behind that target selection, it only contained $\sim 1600$ quasars and was easily supplanted by the SEQUELS survey described in Section 5, which comprised $\sim 21,700$ quasars. Readers interested in an up-to-date description and depiction of the properties of $e B O S S$ quasars as compared to SDSS-I/II/III, should therefore consult Section 5.3 and, in particular, Figures 17 and 18.

The CFHTLS-W3 test survey is detailed in the appendix of Alam et al. (2015). Broadly, an optical selection was applied to SDSSDR8 imaging, restricting to PRIMARY point sources in the (PSF, unextincted) magnitude range $17<r<22$. From this initial sample, objects were targeted for follow-up spectroscopy if they had an $X D Q S O z$ probability of greater than 0.2 of being a quasar at any redshift (i.e., PQSO $(z>0.0)>0.2)$.

Because the CFHT W3 test survey targeted objects regardless of their redshift probability density (all objects with $\operatorname{PQSO}(z>0.0)>0.2)$, the results of the survey could be optimized to better recover quasars in the eBOSS CORE redshift range of $0.9<z<2.2$. One initial outcome of the CFHT W3 test survey, then, was that objects with PQSO $(z>0.0)>0.2$ but $\mathrm{PQSO}(z>0.9)<0.2$ were rarely quasars

\footnotetext{
${ }^{43}$ http://terapix.iap.fr/cplt/oldSite/Descart/summarycfhtlswide.html
}

in the $e B O S S$ redshift range of interest, as demonstrated in Table 1. Further, restricting the redshift range of $e B O S S$ quasar targets to $z>0.9$ is desirable to mitigate losses of (e.g., eBOSS Luminous Red Galaxies targeted at $z<0.9$; c.f. Prakash et al. 2015b) due to fiber collisions between neighboring targets. Therefore, it was decided to focus only on targets with $\mathrm{PQSO}(z>0.9)>0.2$ for $e B O S S$ targeting; we will subsequently restrict our discussion to such targets.

Figure 2 shows the typical positions of $X D Q S O z$ PQSO $(z>0.9)>0.2$ quasars in SDSS colors. To demonstrate the position of $X D Q S O z$-selected quasars in optical color space, we use the large spectroscopically confirmed quasar sample from the DR10 quasar catalog of Pâris et al. (2014). In general, $X D Q S O z$ selects similar regions of color space to SDSS targets from earlier surveys (e.g., Richards et al. 2001), with the majority of the quasar-star separation occuring in the $u g r$ filters.

Whether an $X D Q S O z \operatorname{PQSO}(z>0.9)$ selection alone is sufficient to meet the $e B O S S$ targeting goal of $58 \mathrm{deg}^{-2}$ quasars is investigated in Figure 3, where the sky density of $\mathrm{XDQSOz}$ selected targets as a function of probability threshold is compared to that of confirmed quasars in the requisite CORE redshift range $(0.9<z<2.2$; see Section 2.2). Figure 3 displays three curves that correspond to source densities in the CFHTLS-W3 test program, which can be used to estimate the "true" densities of quasars and targets expected in $e$ BOSS. The lowest (magenta) curve represents all sources in SDSS imaging in the CFHTLS-W3 field that meet the basic CORE cuts (i.e., PRIMARY point sources within the CORE magnitude limits), as a fraction of the total density of $\sim 3330 \mathrm{deg}^{-2}$ such sources. The central (red) curve represents all quasars that were spectroscopically confirmed as part of the CFHTLS-W3 program, as a fraction of the total density of $\sim 135 \mathrm{deg}^{-2}$ such sources. The upper (blue) curve represents all quasars in the specific CORE redshift range of $0.9<z<2.2$ that were spectroscopically confirmed as part of the CFHTLS-W3 program as a fraction of the total density of $\sim 85 \mathrm{deg}^{-2}$ such sources. Because the CFHTLS-W3 program was limited to $\operatorname{PQSO}(z>0.0)>0.2$, the test sample is partially incomplete to quasars that have $\operatorname{PQSO}(z>0.9)<0.2$; such quasars only appear in the CFHTLS-W3 test data due to targeting approaches that did not use $\mathrm{XDQSOz}$ selection. Figure 3 therefore provides best estimates only for PQSO $(z>0.9)>0.2$.

Figure 3 can be used to estimate the total density of quasars and targets that might be expected in eBOSS for different $\operatorname{PQSO}(z>0.9)$ constraints. For example, to estimate the sky density of all quasars at $\mathrm{PQSO}(z>0.9)>0.6$, one would find the corresponding fraction of total $(\sim 0.57)$ and multiply by the total for all quasars $\left(134.3 \mathrm{deg}^{-2}\right)$ to obtain $\sim 77 \mathrm{deg}^{-2}$. The vertical lines in Figure 3 depict the necessary constraints to achieve the requisite $e B O S S$ CORE density of $58 \mathrm{deg}^{-2}$ $0.9<z<2.2$ quasars and the requisite $e B O S S$ target density of $115 \mathrm{deg}^{-2}$ (see Section 2.2). The maximum target density of $115 \mathrm{deg}^{-2}$ is achieved at $\operatorname{PQSO}(z>0.9)>0.45$, which would result in $64.9 \mathrm{deg}^{-2}$ CORE quasars. In actuality, a more relaxed constraint of $\operatorname{PQSO}(z>0.9)>0.2$ is adopted for $e B O S S,{ }^{44}$ which further improves quasar targeting. This relaxed constraint, which is labeled "Adopted cut with IR constraint (see Section 4.1.3)" in Figure 3, was achieved through an additional constraint on mid-IR-optical color (see also Section 4.1.2).

\footnotetext{
${ }^{44}$ Note that this parameter space extends well beyond the effective equivalent cut of $\mathrm{PQSO}(2.2<z<3.5)>0.424$ that was adopted for BOSS.
} 

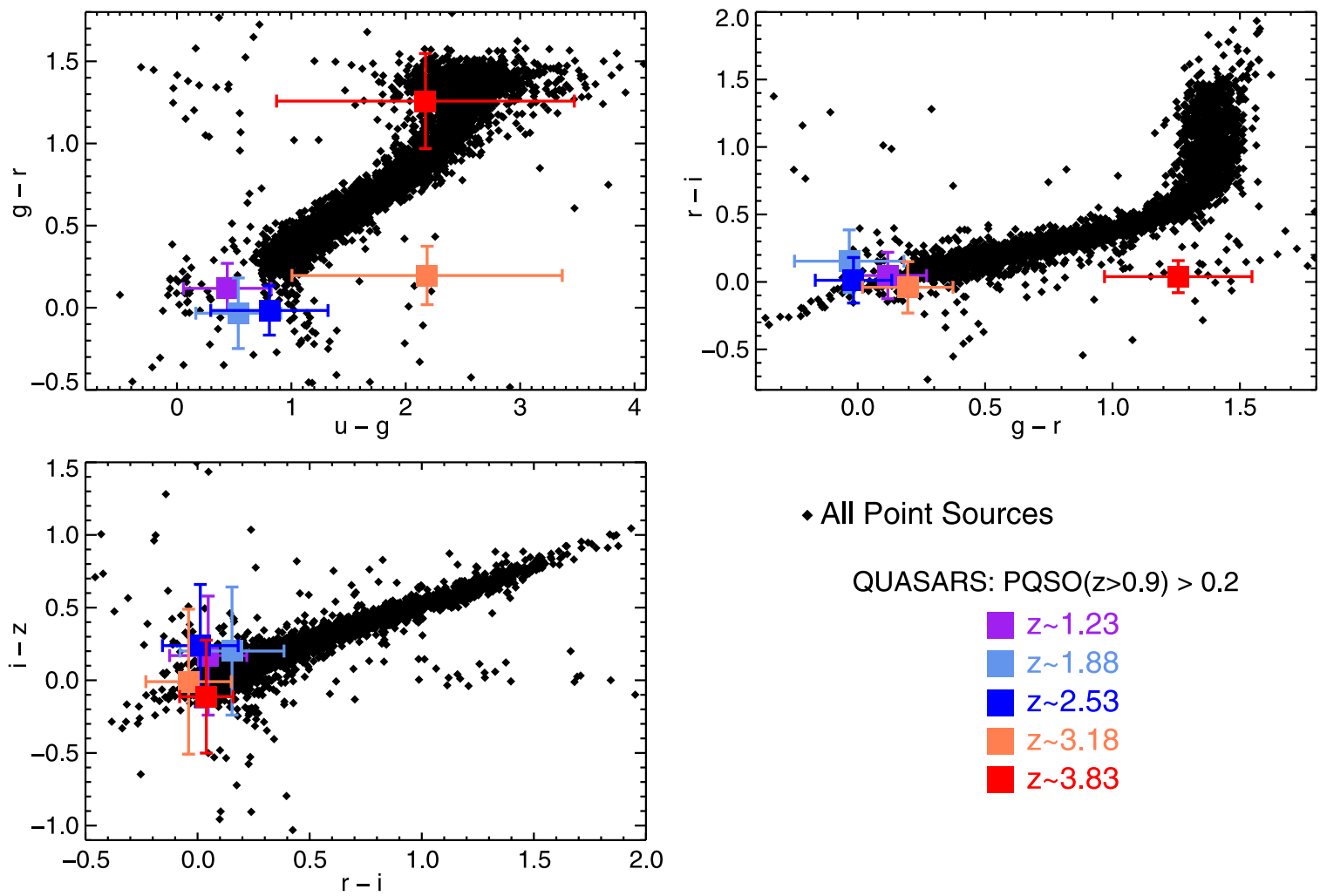

- All Point Sources

QUASARS: $P Q S O(z>0.9)>0.2$

$z \sim 1.23$

$z \sim 1.88$

$z \sim 2.53$

$z \sim 3.18$

$z \sim 3.83$

Figure 2. Position of XDQSOz-selected $\mathrm{PQSO}(z>0.9)>0.2$ quasars in ugriz optical color space (using PSF magnitudes). Black points depict $r<19$ PRIMARY point sources from a randomly chosen SDSS imaging run (5225). The $r<19$ limit is chosen in order to illustrate the position of the stellar locus in SDSS filters; at fainter limits the locus widens considerably (see, e.g., Figures 5 and 6 of Bovy et al. 2011a). Spectroscopically confirmed $\mathrm{PQSO}(z>0.9)>0.2$ quasars from $B O S S$ (DR10; squares) are plotted as a function of redshift, from $z=0.9$ to $z=4.15$ in bins of $\Delta z=0.65$. The error bars indicate the $1 \sigma$ scatter.

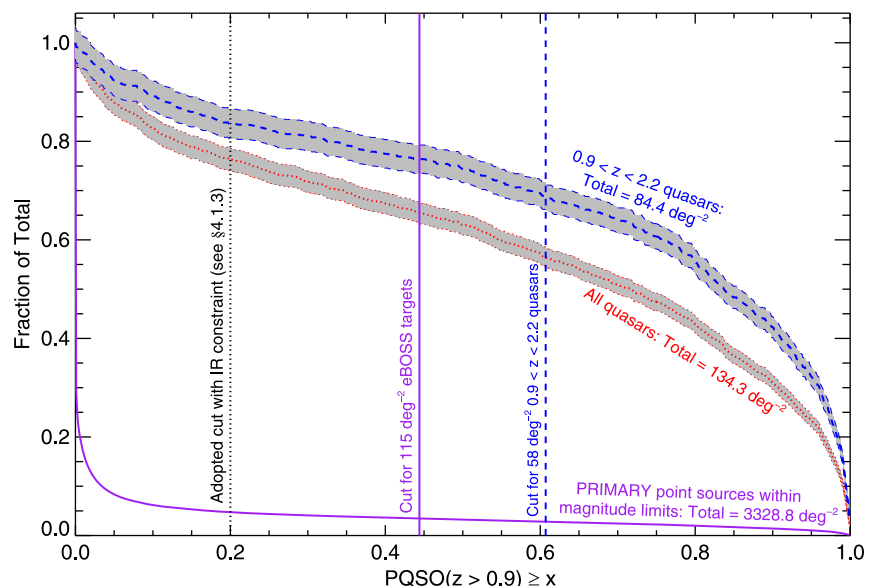

Figure 3. Cumulative sky density of quasars and targets as a function of $z>0.9 \mathrm{XDQSO} z$ probability. The upper curves represent all quasars (red) and $0.9<z<2.2$ quasars (blue) from the CFHTLS-W3 test program. These curves yield an estimate of the completeness of $e B O S S$ to quasars for various $\operatorname{PQSO}(z>0.9)$ constraints. Gray contours illustrate the (Poisson) errors. The lowest curve represents all sources from SDSS imaging in the CFHTLS-W3 test region (magenta). This curve yields an estimate of the necessary fiber budget for $e B O S S$. A quantitative example of how to use the curves to predict quasar and target densities is provided in Section 4.1.1. The vertical lines depict the adopted cut for eBOSS (after also applying an optical-IR color cut; see Section 4.1.3), the cut for the $e B O S S$ requirement of $58 \mathrm{deg}^{-2} 0.9<z<2.2$ quasars, and the cut to assign $<115 \mathrm{deg}^{-2} e B O S S$ fibers (the maximum assignable; see Section 2.2). All samples depicted have been limited to SDSSPRIMARY point sources with FIBER2MAG of $i>17$ and de-extincted PSF magnitudes of $g<22$ or $r<22$ (the initial cuts for the $e$ BOSS CORE).

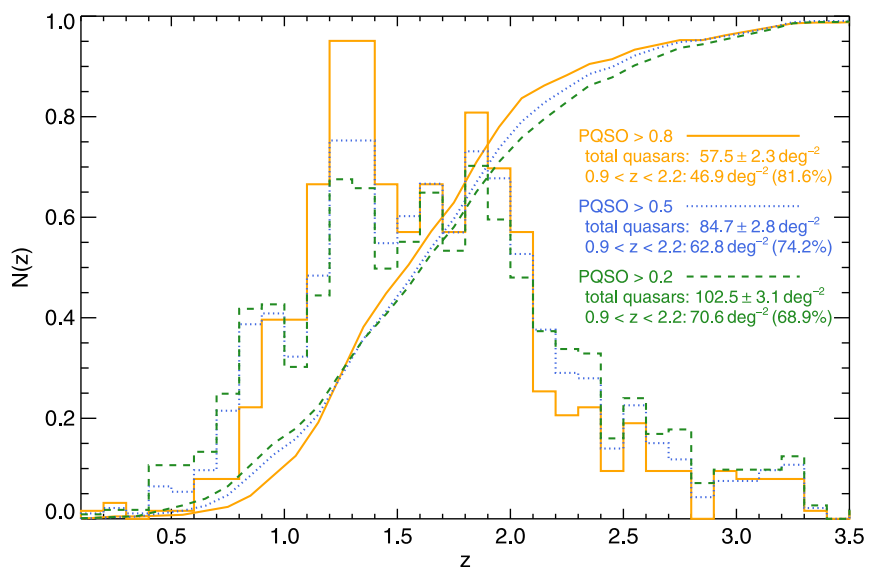

Figure 4. Redshift distribution of spectroscopically confirmed quasars from the CFHTLS-W3 test program. The distributions that peak in the $0.9<z<2.2$ range are the redshift Probability Density Functions (PDFs). The distributions that climb to 1 near $z \sim 3.5$ are cumulative. The distributions for three different cuts on the $z>0.9 \mathrm{XDQSO} z$ probability are depicted; $\mathrm{PQSO}(z>0.9)>0.8$ (orange, solid), $\operatorname{PQSO}(z>0.9)>0.5$ (blue, dotted), and PQSO $(z>0.9)>0.2$ (green, dashed)

Figure 4 depicts how relaxing constraints on $\operatorname{PQSO}(z>0.9)$ to thresholds as low as our adopted $\operatorname{PQSO}(z>0.9)>0.2$ affects the redshift distribution of targeted quasars. The resulting $N(z)$ distributions are broadly similar, but the PQSO $(z>0.9)>0.2$ selection has a tail to $z<0.9$ and contains a smaller fraction of quasars in the CORE target range of 


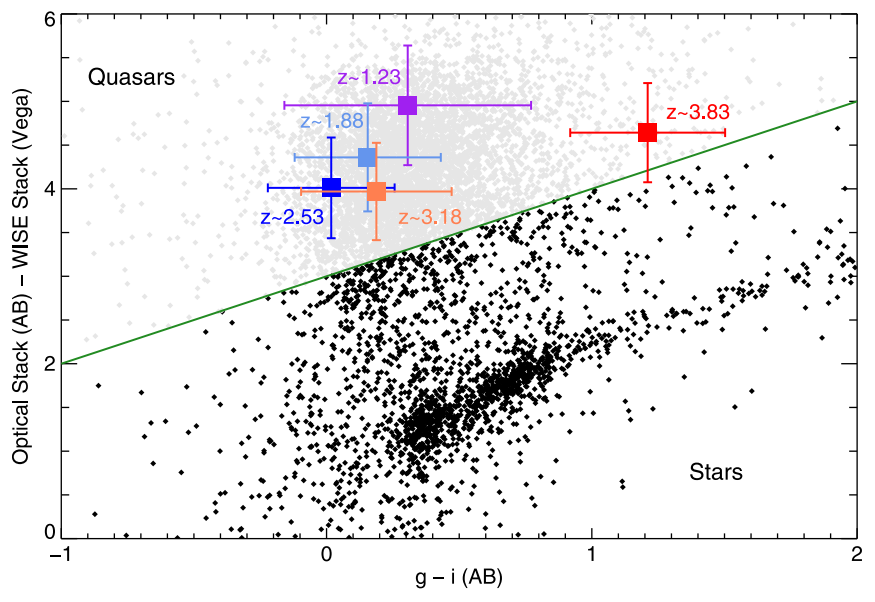

Figure 5. Optical-IR cut (applied to PSF magnitudes) used to define $e B O S S$ CORE quasar targets. The green line depicts the color cut in the $\operatorname{SDSS}\left(f_{g}+0.8 f_{r}+0.6 f_{i}\right) / 2.4$ and $\operatorname{WISE}\left(f_{W 1}+0.5 f_{W 2}\right) / 1.5$ stacks vs. $g-i$ that was used to target quasars as part of the CFHTLS-W3 test program. Quasars of interest to $e B O S S(z \lesssim 3.5)$ generally occupy the region above this line; the stellar locus is a dense region in the lower part of the plot. Black points depict objects with any eBOSS targeting bit set (see Section 4.4) from a randomly chosen SDSS imaging run (5225) limited to $g<22$. Spectroscopically confirmed quasars from BOSS (DR10; squares) are plotted as a function of redshift, from $z=0.9$ to $z=4.15$ in bins of $\Delta z=0.65$. The error bar indicates the $1 \sigma$ scatter.

$0.9<z<2.2$. This drop is more than offset by the PQSO $(z>0.9)>0.2$ selection containing more total quasars (c.f., Figure 3). The peak near $z \sim 1.3$ is likely an artifact of the small sample size in the CFHTLS-W3 test program (c.f., Figure 17). Figure 4 demonstrates that the majority of quasars selected at $\operatorname{PQSO}(z>0.9)>0.2$ remain useful for $e B O S S$ by being in the CORE redshift range of $0.9<z<2.2$. In fact, there is an additional advantage to relaxing the $\mathrm{XDQSOz}$ probability; doing so tends to introduce new quasars at $z>2.1$, while retaining the quasars in the CORE redshift range. Quasars at $z>2.1$ remain useful for the purposes of $e B O S S$ as part of the Ly $\alpha$ sample (see Section 4.2).

\subsubsection{Mid-IR-optical Color Cuts}

Starlight tends to greatly diminish at wavelengths redwards of 1-2 $\mu \mathrm{m}$, making galaxies, and in particular stars, dim in the mid-IR, whereas Active Galactic Nuclei (AGN) have considerable IR emission. Photometric selection techniques based on WISE data can therefore be used to target active galaxies, and such techniques uncover both unobscured and obscured quasars over a range of luminosities (e.g., Stern et al. 2012; Assef et al. 2013; Yan et al. 2013).

Significantly more than half of the objects targeted using mid-IR selection are low-luminosity unobscured AGN at $z<1$ or obscured quasars (e.g., Lacy et al. 2013; Hainline et al. 2014). This makes a pure WISE selection approach imperfect for eBOSS targeting, because objects without an optical spectrum and/or AGN at $z<0.9$ will not typically have utility for the $e B O S S$ CORE goal of targeting $>58 \mathrm{deg}^{-2}$ $0.9<z<2.2$ quasars. WISE remains ideal, however, for removing contaminating stars from $e B O S S$ quasar selection. Figure 5 demonstrates the utility of a WISE-optical color cut in selecting against stars. This color cut is based on stacking optical and WISE fluxes to attain as great a depth as possible. A stack is created from SDSS PSF fluxes according to

$$
\text { Optical Stack }=f_{\mathrm{opt}}=\left(f_{g}+0.8 f_{r}+0.6 f_{i}\right) / 2.4,
$$

and from fluxes in the bluest (and also deepest) WISE bands according to

$$
\text { WISE Stack }=f_{\text {WISE }}=\left(f_{W 1}+0.5 f_{W 2}\right) / 1.5,
$$

where the weights are chosen to roughly yield the highest combined $\mathrm{S} / \mathrm{N}$ for a typical $z<2$ quasar. The sample depicted by black points in Figure 5 represents objects with any eBOSS quasar targeting bit set (see Section 4.4). This sample has been limited to $r>21$ and $g<22$ to illustrate the scatter at the faint end of $e B O S S$, demonstrating the power of the WISE data in filtering stars that other methods target due to these stars' resemblance to quasars in optical colors.

As part of the the CFHTLS-W3 test survey introduced in Section 4.1.1 WISE was photometered at the positions of SDSS PRIMARY sources (see Section 3.2) in the CFHT Legacy survey W3 field. A WISE-SDSS selected sample was created by applying the cut depicted in Figure 5 to these W3-test-field sources;

$$
m_{\mathrm{opt}}-m_{W I S E} \geqslant(g-i)+3,
$$

where $m_{\mathrm{opt}}$ and $m_{\text {WISE }}$ are defined in Equations (1) and (2) after converting the stacked fluxes to magnitudes. ${ }^{45}$ An inclusive stargalaxy separation of objc_type $==6$ or $m_{\mathrm{opt}}-m_{\text {model }}<0.1$, where $m_{\text {model }}$ is the equivalent of Equation (1) but for $S D S S$ model magnitudes, was adopted. This is inclusive in the sense that objc_type $==6$ corresponds to a star-galaxy separation of psfMag - modelMag $\leqslant 0.145$ (as also discussed further in Section 4), but based on SDSS fluxes in all bands, not just the bands stacked in $m_{\text {opt }}$. In addition, magnitude limits of $17<m_{\text {opt }}<22$ were enforced. Finally, an optical color cut of $g-i<1.5$ was applied in an attempt to excise the highest redshift quasars (this cut is not obvious in Figure 5 because other programs in the CFHTLS-W3 test program repopulated this parameter space). The squares with error bars in Figure 5 depict the typical range of colors of spectroscopically confirmed quasars in different redshift bins. The separation of these points from the green line suggests that WISE is robust for quasar selection across the CORE redshift range of $0.9<z<2.2$.

Figure 6 demonstrates whether a WISE-optical cut of $m_{\mathrm{opt}}-m_{\text {WISE }} \geqslant(g-i)+x$ is sufficient, in isolation, to meet the $e B O S S$ targeting goal of $58 \mathrm{deg}^{-2} 0.9<z<2.2$ quasars (contingent on our additional restrictive cuts to the W3-testfield targets, such as $g-i<1.5$ ). Figure 6 is an exact analog of Figure 3, and a detailed description of how these figures can be interpreted is provided in Section 4.1.1. Figure 6 implies that a cut of about $m_{\mathrm{opt}}-m_{\text {WISE }} \geqslant(g-i)+4.25$ is necessary to meet the requisite $e B O S S$ target density of $115 \mathrm{deg}^{-2}$ and that, therefore, only $34.1 \mathrm{deg}^{-2}$ CORE quasars could be obtained with a WISE-optical selection alone. As discussed further in Section 4.1 .3 , by combining $X D Q S O z$ selection with WISE eBOSS we could use the "Adopted cut..." plotted in Figure 6. This relaxed cut does achieve eBOSS targeting goals.

Figure 7 demonstrates that relaxing cuts on $x$ in the function $m_{\mathrm{opt}}-m_{\text {WISE }} \geqslant(g-i)+x$ does not strongly affect the

\footnotetext{
${ }^{45}$ This cut was also eventually used for $e$ BOSS CORE quasar target selection.
} 


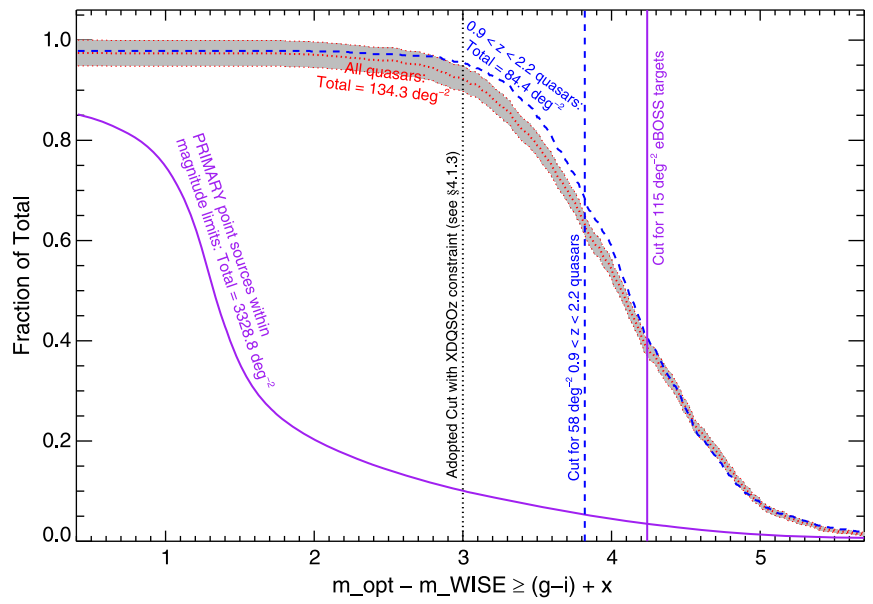

Figure 6. As for Figure 3, but for the adopted WISE-optical cut. The $x$-axis depicts the number of sources for a cut of $\geqslant x$, where $x$ is defined by $\left(m_{\mathrm{opt}}-m_{\text {WISE }}\right)=(g-i)+x$ and $m_{\mathrm{opt}}$ and $m_{\text {WISE }}$ are the magnitudes from the optical and WISE stacks. The gray (Poisson) error contours have been omitted from the blue curve for visual clarity, but are comparable to the errors on the red curve. All samples depicted have been limited to SDSS PRIMARY point sources with FIBER2MAG of $i>17$ and de-extincted PSF magnitudes of $g<22$ or $r<22$ (the initial cuts for the eBOSS CORE). Because the CFHTLS-W3 program was limited to $\left(m_{\mathrm{opt}}-m_{\text {WISE }}\right)>(g-i)+3$ the test sample is partially incomplete to quasars for $x<3$. This figure can be used to estimate target densities in a similar manner to Figure 3.

redshift distribution of targeted quasars. This figure shows that $65 \%-70 \%$ of quasars selected by this WISE-SDSS cut are in the CORE redshift range, regardless of the value of $x$. Overall, there is less variation in the eBOSS CORE $0.9<z<2.2$ redshift distribution with $x$ as compared to the variation in Figure 4, because the WISE-optical cut has less power to discriminate redshift as compared to ugriz over most of the CORE range (c.f. Figure 5). Instead of augmenting the CORE quasar range, relaxing $x$ tends to expand the fraction of quasars at about $z>2$. This outcome is desirable, given that $z>2.1$ quasars can be used as part of the $e B O S S$ Ly $\alpha$ sample (see Section 4.2).

By redshifts of $z \sim 6$, about half of quasars are not detected in the WISE $W 1$ and $W 2$ bands (Blain et al. 2013). In addition, a $10 \sigma$ detection in WISE W2 is equivalent to $i \sim 19.8$ (Stern et al. 2012), which may not detect all quasars to the effective $e B O S S$ limits of $r \sim 22$. Thus it is worth investigating whether the WISE data photometered for eBOSS targeting (see Section 3.2) are sufficiently deep for our purposes. Figure 8 addresses this issue by plotting known $D R 10$ quasars as a function of $\mathrm{S} / \mathrm{N}$ in our WISE stack $\left(m_{\text {WISE }}\right)$. The stack depth is sufficient to identify $90 \%$ of $0.9<z<2.2$ BOSS quasars at a S/N of 2 in the stack to $r<21.9$. Although the depth of WISE becomes limiting near $r \sim 22$ for eBOSS CORE quasars, about $93 \%$ of $0.9<z<2.2$ BOSS quasars would be selected by our WISEoptical cut; this is because of the combined effect that few quasars are both blue in $g-i$ and faint in WISE.

\subsubsection{Combined Mid-IR and Optical Selection}

After analyzing our CFHTLS-W3 test data (as outlined in Sections 4.1.1 and 4.1.2) it became clear that the overall number of CORE quasars targeted at the $e B O S S$ fiber density could be increased by combining an $X D Q S O z$ probability limit with a WISE-optical cut. It was possible to only partially study the XDQSOz probability and WISE-optical cut beyond the

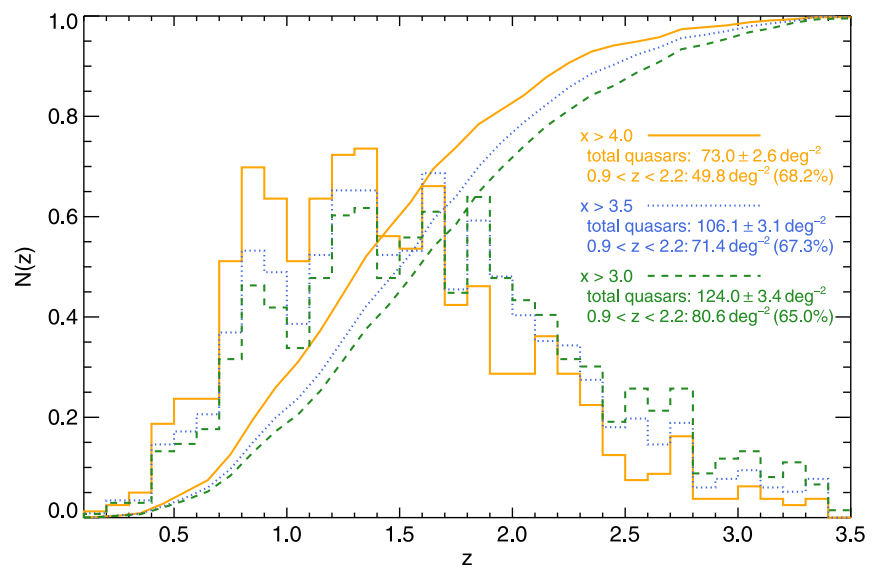

Figure 7. As for Figure 4, but for the adopted WISE-optical cut. The distributions for three different cuts on $x$ are depicted, where $x$ is defined by $\left(m_{\mathrm{opt}}-m_{W I S E}\right)=(g-i)+x$ and $m_{\mathrm{opt}}$ and $m_{W I S E}$ are the magnitudes from the optical and WISE stacks. These cuts are $x>4.0$ (orange, solid), $x>3.5$ (blue, dotted), and $x>3.0$ (green, dashed).

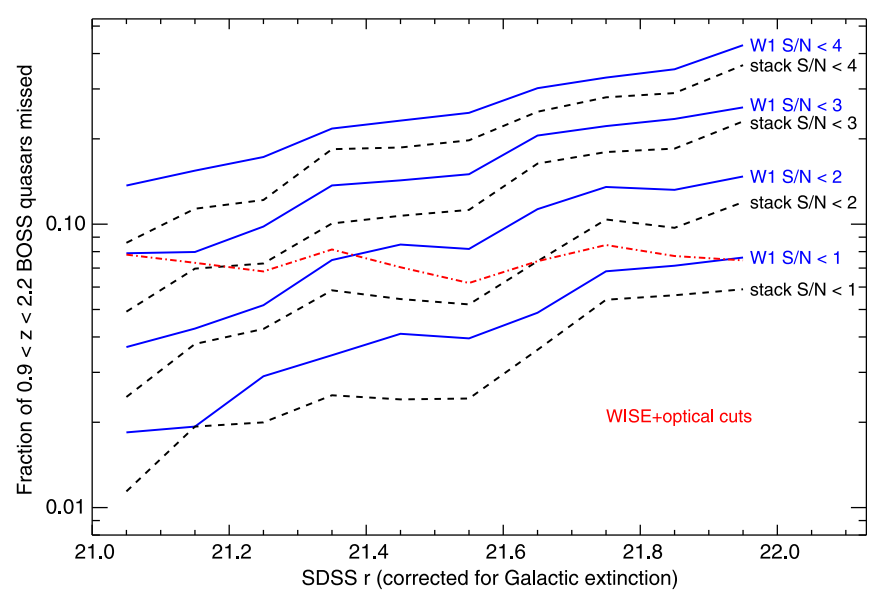

Figure 8. Fraction of $0.9<z<2.2$ (DR10) BOSS quasars that are missed as a function of WISE signal-to-noise ratio in the $W 1$ band (blue solid line) and in the stack of $\left(f_{W 1}+0.5 f_{W 2}\right) / 1.5$ that is actually used in $e B O S S$ CORE quasar selection (black dashed line). The red (dotted-dashed) line displays the fraction of such quasars missed by the overall eBOSS CORE quasar target selection.

limits to which they had been tested in the CFHTLS-W3 program-using those $X D Q S O z$-selected quasars that failed the $W I S E$-optical cut and vice versa. Because the combination of the two original test cuts exceeded $e B O S S$ goals, however, it was decided to proceed with an $e B O S S$ CORE quasar target selection corresponding to both

$$
\begin{gathered}
\operatorname{PQSO}(z>0.9)>0.2 \quad \text { and } \\
m_{\mathrm{opt}}-m_{\text {WISE }} \geqslant(g-i)+3 .
\end{gathered}
$$

The "Adopted cut..." lines in Figures 3 and 6 demonstrate that in combination these constraints easily achieve the $e B O S S$ CORE goal of $58 \mathrm{deg}^{-2} 0.9<z<2.2$ quasars. It turns out that the combined $X D Q S O z$-and-WISE-optical constraints that correspond to these adopted cuts require close to the maximum eBOSS quasar target density of $115 \mathrm{deg}^{-2}$ (see Section 2.2) and achieve an overall density of $\sim 70 \mathrm{deg}^{-2} 0.9<z$ $<2.2$ quasars. The expected eBOSS CORE quasar density arising from these constraints is explored in more detail in Section 5.1. 


\subsection{Broad Overview of the Ly $\alpha$ Quasar Sample}

The goal of $e B O S S \operatorname{Ly} \alpha$ quasar targeting is to compile as large a sample of new $z>2.1$ quasars as possible using the remaining available fibers that were not allocated to other $e B O S S$ targets. The $e B O S S \operatorname{Ly} \alpha$ sample is not required to be homogeneously selected; it is therefore targeted using several different selection algorithms and sources of imaging-even imaging that only partially covers the eBOSS footprint.

The majority of new $e B O S S$ Ly $\alpha$ quasars are targeted using two techniques. First, the CORE sample described in Section 4.1 is a source of new Ly $\alpha$ quasars, because its selection contains no requirement to intentionally remove $z>2.1$ quasars. Second, a variability selection is used to target additional Ly $\alpha$ quasars. The CORE and variability-selected samples each select $\sim 5 \mathrm{deg}^{-2}$ new Ly $\alpha$ quasars, with only $\sim 1.5 \mathrm{deg}^{-2}$ in common (see also Table 4 in Section 5.2). The variability-selected targets undergo a different set of initial flag and flux cuts as compared to other target classes (see Section 4.2.1).

eBOSS uses two additional techniques to target more Ly $\alpha$ quasars and acquire more signal in the Ly $\alpha$ Forest. First, all previously unidentified sources within $1^{\prime \prime}$ of a radio detection in the FIRST survey (Becker et al. 1995; Helfand et al. 2015) are targeted. Then, quasars that had low $\mathrm{S} / \mathrm{N}$ spectra in BOSS are retargeted. The target categories specific to Ly $\alpha$ selection are detailed in the following and are summarized in Section 4.4.

\subsubsection{Variability Selection}

Time-domain photometric measurements can exploit quasars' intrinsic variability in order to distinguish them from stars of similar colors (e.g., van den Bergh et al. 1973; Hawkins 1983; Cimatti et al. 1993; Rengstorf et al. 2004a, 2004b; Claeskens et al. 2006; Sesar et al. 2007; Kozłowski et al. 2010; MacLeod et al. 2010; Schmidt et al. 2010; PalanqueDelabrouille et al. 2011, 2013a, 2015). The time-variability of astronomical sources can be described using the "structure function," a measure of the amplitude of the observed variability as a function of the time delay between two observations (e.g., Cristiani et al. 1996; Giveon et al. 1999; Vanden Berk et al. 2004; Rengstorf et al. 2006). This function can be modeled as a power law parameterized in terms of $A$, the mean amplitude of the variation on a one-year timescale (in the observer's reference frame), and $\gamma$, the logarithmic slope of the variation amplitude with respect to time (Schmidt et al. 2010). With $\Delta m_{i j}$ defined as the difference between the magnitudes of the source at time $t_{i}$ and $t_{j}$, and assuming an underlying Gaussian distribution of $\Delta m$ values, the model predicts an evolution of the variance $\sigma^{2}(\Delta m)$ with time according to

$$
\sigma^{2}(\Delta m)=\left[A\left(\Delta t_{i j}\right)^{\gamma}\right]^{2}+\left(\sigma_{i}^{2}+\sigma_{j}^{2}\right)
$$

where $\sigma_{i}$ and $\sigma_{j}$ are the imaging errors at time $t_{i}$ and $t_{j}$. Quasars should lie at high $A$ and $\gamma$; non-variable stars near $A=\gamma=0$ and variable stars should have $\gamma$ near 0 even if $A$ is large. In addition, variable sources (whether stars or quasars) are expected to deviate greatly from a model with constant flux. This deviation is quantified by computing the $\chi^{2}$ of the fit of the light curve compared to a constant-flux model.

Using customized PTF $R$-band stacks (see section Section 3.3), light curves are built for all PTF sources. The $P T F$ sources are matched to SDSS imaging catalogs, and the

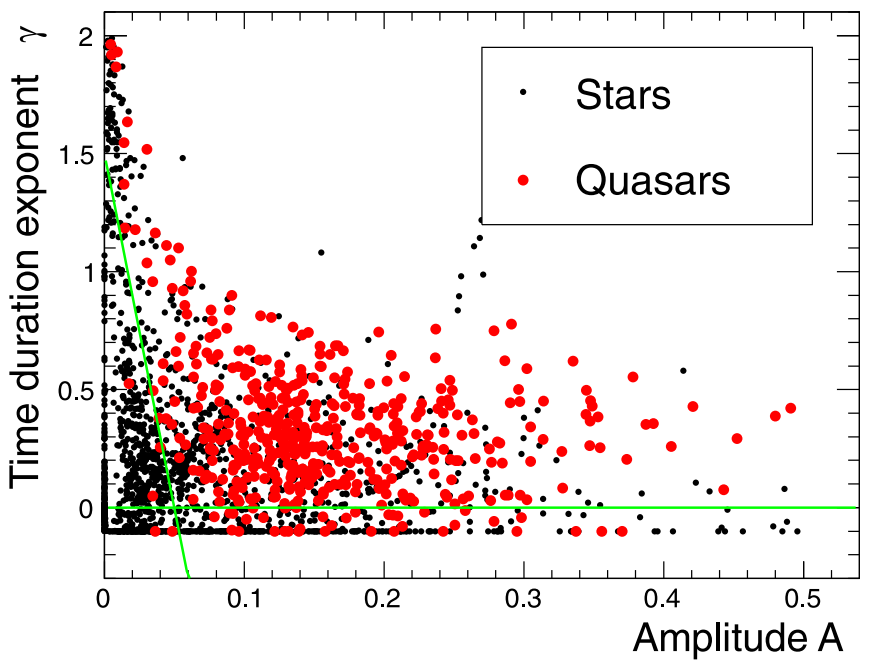

Figure 9. Structure function parameters for six-epoch $R$-band light curves from $P T F$. Quasars (red) and stars (black), whether variable or non-variable, populate distinct regions of the $\gamma-A$ plane. Stars are a subsample of 1500 random point-like objects delimited in Equatorial Coordinates by $52^{\circ}<\delta_{\mathrm{J} 2000}<54^{\circ}$ and $211^{\circ}<\alpha_{\mathrm{J} 2000}<216^{\circ}$. Quasars are the previously identified quasars (mostly from BOSS) in the same field.

selection is restricted to SDSS PRIMARY point sources. With the PTF light curves in hand, all additional cuts are then applied using SDSS imaging information. SDSS cuts of $g<22.5$ and $r>19$ are then applied. When SDSS $r$-band data are available, the $R$-band $P T F$ light curve, adjusted to $S D S S r$, is extended to include the SDSS fluxes. These PTF +SDSS light curves typically contain three to four PTF "coadded epochs," where each PTF coadded epoch is obtained by coadding the exposures within a given PTF observational season. The number of exposures in each season varies from $\sim 10$ to a few dozen for typical fields.

Because the density of PTF images varies across the sky, so does the efficiency of the variability-based selection. To account for this, the thresholds of the variability cuts are adapted as a function of position in order to reach an average target density of $\sim 20 \mathrm{deg}^{2}$ across the eBOSS footprint. Constraints of $5.0<\chi^{2}<200.0$ for combined PTF $+S D S S$ measurements are typically necessary; smaller $\chi^{2}$ values are obtained for non-variable sources, while larger values often signify artifacts. The parameters of the variability structure function are forced to lie in the parameter space bounded by $\gamma>0$ and $\gamma>-30 A+1.5$, as illustrated by the green lines in Figure 9. Tighter $\chi^{2}$ cuts are applied to light curves for which the variability parameters $A$ and $\gamma$ cannot be computed reliably, such as light curves with fewer than three $P T F$ epochs.

To maximize the efficiency of quasar selection, the variability selection is complemented by loose color cuts designed to reject stars. Cuts of $c_{3}<1.4-0.55 \times c_{1}$ and $c_{3}<0.3-0.1 \times c_{1}$ are imposed, where

$$
\begin{aligned}
& c_{1}=0.95(u-g)+0.31(g-r)+0.11(r-i) \\
& c_{3}=-0.39(u-g)+0.79(g-r)+0.47(r-i),
\end{aligned}
$$

as defined in Fan (1999). In these equations, ugri are PSF magnitudes measured in the SDSS imaging. This color cut is illustrated in Figure 10, where the regions above the red and green lines are rejected.

Finally, a region in color space mostly populated by bright variable stars, that passes both the color and the variability cuts, 


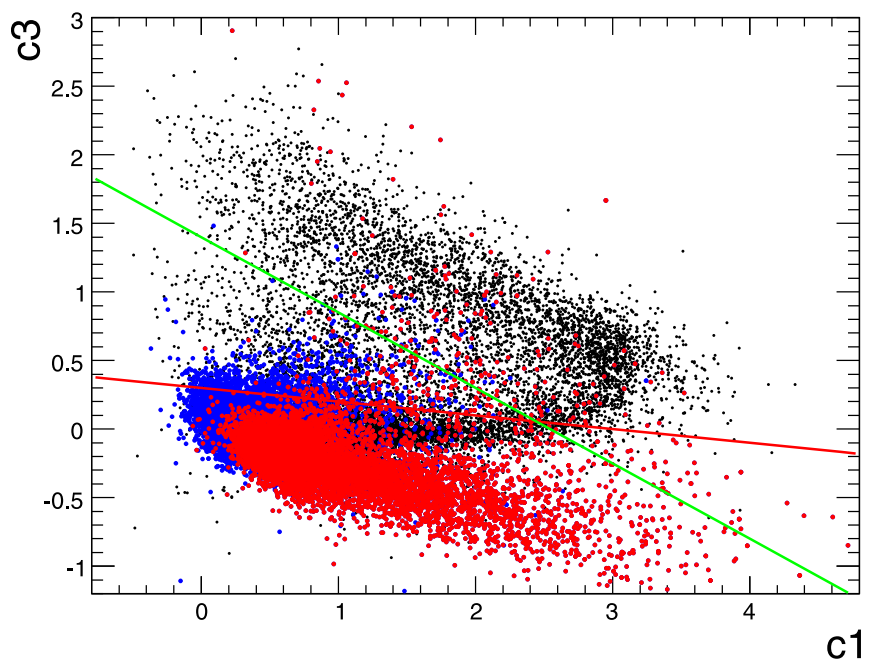

Figure 10. Adopted loose color cut designed to reject stars. Black, blue, and red points represent stars, $z<2.1$ quasars, and $z>2.1$ quasars, respectively. The colors of each set of objects are taken from the SDSS Catalog Archive Server. Stars are obtained from a $7.5 \mathrm{deg}^{2}$ region delimited by $357^{\circ}<\alpha_{\mathrm{J} 2000}<360^{\circ}$ and $-1.25<\delta_{\mathrm{J} 2000}<1.25$ (i.e., they represent a random sample of point-like objects). Quasars are a subsample of spectroscopically confirmed sources from the SDSS surveys.

is removed. These stars are apparent in the top panel of Figure 11 -but are clearly absent in the lower panel, which depicts known quasars. These contaminating variable stars are removed by rejecting sources that lie in the color box $0.85<c_{1}<1.35$ and $c_{3}>-0.2$ if they are brighter than $r=20.5$. This cut is not applied to fainter sources.

\subsubsection{Reobservation of BOSS Quasars}

The mean density of Ly $\alpha$ quasars in BOSS (once Broad Absorption Line quasars are removed) is $\sim 15 \mathrm{deg}^{-2}$. Roughly $60 \%$ of these quasars have an $\mathrm{S} / \mathrm{N}<3$, thus reducing their utility for tracing large-scale structure. Here, $\mathrm{S} / \mathrm{N}$ is defined as the mean $\mathrm{S} / \mathrm{N}$ per Ly $\alpha$ Forest pixel measured over the restframe wavelength range of $1040 \AA<\lambda<1200 \AA$. With the exception of BOSS spectra that have $\mathrm{S} / \mathrm{N} \mathrm{pixel}^{-1}=0$ (signifying an observational error) quasars with $0 \leqslant$ $\mathrm{S} / \mathrm{N}_{\text {pixel }}{ }^{-1}<0.75$ do not contribute as much to the Forest signal as placing a fiber on a new quasar target, so such quasars are not worth reobserving. Within eBOSS, BOSS quasars are therefore targeted if they lie in the $e B O S S$ footprint and have $0.75 \leqslant \mathrm{~S} / \mathrm{N}_{\text {pixel }}{ }^{-1}<3$ or $\mathrm{S} / \mathrm{N}$ pixel ${ }^{-1}=0$. The density of these targets varies over the $e B O S S$ footprint from $\sim 6 \mathrm{deg}^{-2}$ to $\sim 10 \mathrm{deg}^{-2}$, depending upon the underlying density of BOSS Ly $\alpha$ quasars.

\subsubsection{Radio Selection}

$e B O S S$ also targets all SDSS point sources that are within $1^{\prime \prime}$ of a radio detection in the 13 June 2005 version $^{46}$ of the FIRST point source catalog (Becker et al. 1995; Helfand et al. 2015). The density of such sources (that are not already included in another target class) is low $\left(<1 \mathrm{deg}^{-2}\right)$, and these additional targets are expected to identify some previously unknown highredshift quasars.

\footnotetext{
${ }^{46}$ http://sundog.stsci.edu/first/catalogs/readme_13jun05.html
}
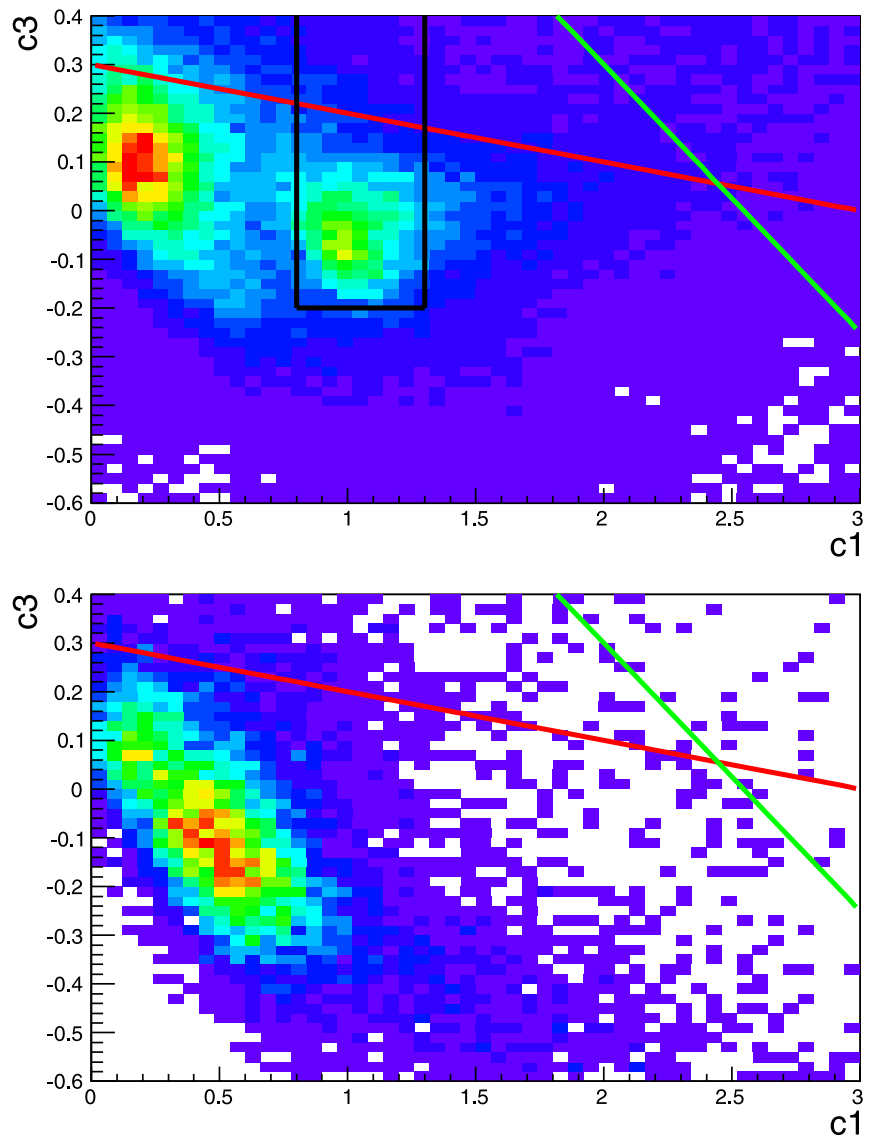

Figure 11. $c_{1}-c_{3}$ color plots for sources passing the variability criteria defined in Section 4.2.1. The upper panel depicts all objects: the two peaks correspond to quasars (left-most density peak) and bright variable stars (rightmost density peak). The lower panel shows previously known quasars only (mostly $z>2.1$ quasars from $B O S S$ ). The contaminating population in the top plot is variable stars that are removed with a dedicated set of color cuts illustrated by the black box (see Section 4.2.1 for more details).

\subsection{Additional Cuts}

SDSS imaging includes a great deal of metadata ${ }^{47}$, and, notably, contains flags (in the form of bitmasks) that can be used to characterize photometric quality. ${ }^{48}$ Initially, eBOSS adopts a set of obvious and necessary cuts on SDSS imaging parameters. The target selection is restricted to PRIMARY sources in the $S D S S$ to avoid duplicate sources. Targets are cut on (de-extincted) PSFMAG to near the limits of SDSS imaging, in part driven by the necessary exposure times to obtain spectra of reasonable $\mathrm{S} / \mathrm{N}$. These limits are $g<22$ or $r<22$ for CORE quasars and $g<22.5$ for the Ly $\alpha$ quasar sample, which can be more speculative and inhomogeneous in its selection. A bright limit of FIBER2MAGi $>17$ is adopted for all $e B O S S$ targets to prevent light leaking between adjacent fibers (see Dawson et al. 2015). Quasars selected by variability and intended purely for Ly $\alpha$ studies have a more restrictive brightend cut of $r>19$, because there are few high-redshift quasars brighter than $r=19$. Finally, the restriction that quasar targets must be unresolved in imaging (objc_type $==6$ ) is imposed. This is necessary because at fainter magnitudes extended sources begin to dominate SDSS imaging, and at $r>21.2$ there are three times as many objc_type $==3$

\footnotetext{
47 E.g., see Tables 5, 6, 8 and 9 of Stoughton et al. (2002).

48 See Table 9 of Stoughton et al. (2002).
} 


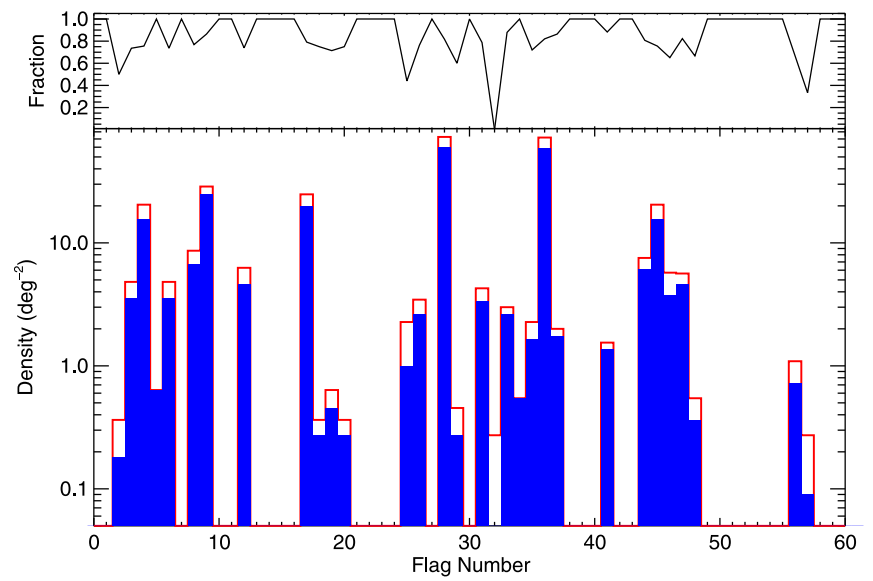

Figure 12. Sky density of quasars and targets removed by a specific SDSS flag cut. Flag numbers 0-31 correspond to the 32 bits in the SDSS objc_flags bitmask and flag numbers $32-63$ are the 32 bits in the SDSS objc_flags 2 bitmask. The final three bits in objc_flags 2 do not correspond to an imaging flag. The red (empty) histogram is the density of targets discarded from the CFHTLS-W3 test data and the blue (filled) histogram is the density of genuine $z>0.9$ quasars discarded by the same flag cut. In the upper panel we display the ratio of the two histograms, which is the fraction of targets discarded that would be useful quasars for $e B O S S$.

(extended) sources as objc_type $==6$ (point-like) sources. Targeting extended sources would greatly increase the $e B O S S$ fiber budget, while recovering few $z>0.9$ quasars.

Our CFHTLS-W3 test program (outlined in Section 4.1.1) had relaxed limits on star-galaxy separation and magnitude, meaning that it is possible to show that our basic flag cuts for eBOSS quasar targeting represent sensible choices. Adopting the selection outlined in Section 4.1.3, a cut on objc_type $==6$ discards only $4.6 \%$ of quasars but requires $3.5 \times$ fewer fibers. Enforcing faint limits of $g<22$ or $r<22$ discards $5.8 \%$ of quasars but requires $11.5 \times$ fewer fibers.

Typically, previous SDSS quasar targeting algorithms (Richards et al. 2002; Ross et al. 2012) have employed additional constraints on image quality to reduce spurious targets. Given that the CFHTLS-W3 test program did not adopt strict flag cuts, it could be used to assess which flag cuts might be worthwhile for $e B O S S$ targeting (see Figure 12). A range of individual SDSS flag cuts are plotted in Figure 12, which demonstrates that there are essentially no SDSS flags that discard targets without also discarding useful $z>0.9$ quasars. The one exception is the DEBLENDED_AS_MOVING flag (number 32), which does not obviously discard quasars, but which only saves $0.3 \mathrm{deg}^{-2}$ targets. In addition to the results in Figure 12, we also tested numerous standard combinations of flags used by other SDSS quasar targeting algorithms, such as the INTERP_PROBLEMS and DEBLEND_PROBLEMS combinations outlined in the appendices of Bovy et al. (2011a) and Ross et al. (2012). In no case did we find a flag combination that removed significant numbers of targets without also discarding useful quasars. We do not study why the $S D S S$ image quality flags have limited utility for $e B O S S$ targetingspeculatively the flags may become less meaningful near the faint limits of SDSS imaging and/or our incorporation of WISE data may ameliorate $S D S S$ artifacts. In any case, based on this analysis and the fact that the basic eBOSS selection already achieves the requisite target density, we make no additional SDSS flag cuts.
Table 2

eBOSS Quasar Targeting Bits and their Numerical Equivalents

\begin{tabular}{llll}
\hline \hline Bit & Name & Bit & Name \\
\hline 0 & DO_NOT_OBSERVE & & \\
10 & QSO_EBOSS_CORE & 15 & QSO_BAD_BOSS \\
11 & QSO_PTF & 16 & QSO_BOSS_TARGET \\
12 & QSO_REOBS & 17 & QSO_SDSS_TARGET \\
13 & QSO_EBOSS_KDE & 18 & QSO_KNOWN \\
14 & QSO_EBOSS_FIRST & 19 & DR9_CALIB_TARGET \\
\hline
\end{tabular}

It is likely that certain regions of the SDSS imaging will have to be masked further for quasar clustering analyses, due to areas around bright stars (both in WISE and SDSS imaging) or bad imaging fields, for example (see Ross et al. 2011, and Section 6). For instance, due to how the SDSS geometry was initially defined for "uber-calibration," small overlap regions $\left(\sim 1 \mathrm{deg}^{2}\right)$ in SDSS run 752 are misaligned between SDSS and our WISE photometering. Such regions do not have a major impact on target homogeneity, however, and may differ for different $e B O S S$ target classes, so such geographic areas will be masked post-facto, depending on a specific science purpose. One set of regions that was masked a priori for BOSS quasar targeting corresponded to bad $u$-columns (e.g., see Figure 1 of White et al. 2012). Specifically testing target density in areas with bad SDSS u-columns did not suggest they have greatly different eBOSS CORE target densities $\left(\sim 116-118 \mathrm{deg}^{-2}\right.$ versus the average of $\sim 115 \mathrm{deg}^{-2}$ for the typical survey area), however, so bad $u$-columns are not specifically masked a priori for $e B O S S$ targeting.

In general, the only large geographic areas that should certainly not be photometric in SDSS imaging are regions with catastrophic values of IMAGE_STATUS. ${ }^{49}$ For eBOSS CORE quasar targeting, we avoid all areas with IMAGE_STATUS set to BAD_ROTATOR, BAD_ASTROM, BAD_FOCUS, SHUTTERS, FF_PETALS, DEAD_CCD, or NOISY_CCD in any filter. Quasars targeted on the basis of their variability in PTF for Ly $\alpha$ studies do not undergo cuts on IMAGE_STATUS because there is no requirement for Ly $\alpha$ quasars to be selected homogeneously. The full set of flag cuts eventually adopted is outlined succinctly in Figure 1.

\subsection{Targeting Bits}

The tests summarized in Sections 4-4.3 provide sufficient information to justify the choices made to target quasars in $e B O S S$. This section provides an outline of how the eBOSS targeting bits directly correspond to the specified choices. A visual representation of the overall targeting algorithm is also provided in Figure 1. Unless otherwise specified, each target class is derived from the imaging outlined in Section 3 and undergoes the basic flag cuts outlined in Section 4.3 (PRIMARY, objc_type $==6$, magnitude cuts, and good IMAGE_STATUS). The numerical value of each $e B O S S$ quasar targeting bit is listed in Table 2 . The density and success rate of each class of target is described further in Section 5.

\subsubsection{QSO_EBOSS_CORE}

Quasars that comprise the main eBOSS CORE sample are assigned the QSO_EBOSS_CORE bit. The main goal of the

\footnotetext{
$\overline{49}$ http://www.sdss3.org/dr8/algorithms/bitmask_image_status.php
} 
CORE sample is to obtain $>58 \mathrm{deg}^{-2} 0.9<z<2.2$ quasars (assuming an exactly $7500 \mathrm{deg}^{2}$ footprint for $e B O S S$ ). We make no attempt to limit the upper end of the CORE redshift range, meaning that the CORE also selects $z>2.1$ quasars that have utility for Ly $\alpha$ Forest studies. Quasars in the CORE are selected by $X D Q S O z$ and WISE as described in Section 4.1.3.

\subsubsection{QSO_PTF}

Quasars intended for Ly $\alpha$ Forest studies typically do not have to be selected in a uniform manner. This freedom allows variability selection to be applied to inhomogeneous imaging in order to target additional $z>2.1$ quasars for $e B O S S$. The QSO_PTF bit indicates such quasars, which have been selected using multi-epoch imaging from the Palomar Transient Factory. PTF targets undergo slightly different initial cuts to other quasar target classes; they are limited in magnitude to $r>19$ and $g<22.5$ and are observed in areas with bad IMAGE_STATUS. These choices are justified in Section 4.3. PTF quasars are selected as described in Section 4.2.1.

\subsubsection{QSO_REOBS}

Quasars previously confirmed in BOSS that are of reduced (but not prohibitively low) $\mathrm{S} / \mathrm{N}$ have decreased utility for $\mathrm{Ly} \alpha$ Forest studies. In addition, high probability BOSS quasar targets that have zero spectral $\mathrm{S} / \mathrm{N}$ in BOSS are likely to have been spectroscopic glitches. The QSO_REOBS bit signifies quasars that were measured to have $0.75 \leqslant \mathrm{~S} / \mathrm{N} \mathrm{pixel}^{-1}<3$ or $\mathrm{S} / \mathrm{N} \mathrm{pixel}^{-1}=0$ in BOSS. Quasars are selected for reobservation as described in Section 4.2.2.

\subsubsection{QSO_EBOSS_KDE}

The QSO_EBOSS_KDE bit has been discontinued for $e$ BOSS but formed part of the targeting for SEQUELS (see Section 5.1). Targets that had the QSO_EBOSS_KDE bit set in SEQUELS were drawn from the Kernel Density Estimation catalog of Richards et al. (2009b) and had uvxts $==1$ set within that catalog. Because the QSO_EBOSS_KDE bit is discontinued, the origin of this target class is not described further in this paper.

\subsubsection{QSO_EBOSS_FIRST}

Powerful radio-selected quasars can be detected by FIRST at $z>2.1$ and can therefore have utility for Ly $\alpha$ Forest studies. The QSO_EBOSS_EIRST bit indicates quasars that are targeted because they have a match in the FIRST radio catalog, as described in Section 4.2.3.

\subsubsection{QSO_BAD_BOSS}

Some likely quasars with spectroscopy obtained as part of $B O S S$ have uncertain classifications or redshifts upon visual inspection. Such objects are designated as QSO? or QSO_Z? in DR12Q (c.f. Pâris et al. 2014). The QSO_BAD_BOSS bit signifies such objects to ensure that ambiguous BOSS quasars are always reobserved, regardless of which other targeting bits are set. Prior to 4 November 2014 (effectively prior to the eboss 6 tiling; see Dawson et al. 2015) a close-to-final but preliminary version of $D R 12 Q$ was used to define this sample, but as of eboss6, the final sample of $D R 12 Q$ was used to define the QSO_BAD_BOSS bit. This change effectively means that a small number of quasars with ambiguous BOSS spectra may not have been reobserved prior to eboss 6 .

\subsubsection{QSO BOSS TARGET}

In an attempt to reduce the overall target density, eBOSS quasar targeting does not retarget any objects with good spectra from BOSS unless otherwise specified. The QSO_BOSS_TARGET bit is set to indicate such objects. We define an object as having good BOSS spectroscopy if it appears in the file of all spectra that have been observed by $B O S S,{ }^{50}$ and if it does not have either LITTLE_COVERAGE or UNPLUGGED set in the ZWARNING bitmask (see Table 3 of Bolton et al. 2012).

\subsubsection{QSO_SDSS_TARGET}

eBOSS quasar targeting will not retarget objects with good pre-BOSS spectra from the SDSS (i.e., spectra from prior to DR8). The QSO_SDSS_TARGET bit is set to indicate such objects. A "good" spectrum is defined using LITTLE_COVERAGE and UNPLUGGED as for the QSO_BOSS_TARGET bit. SDSS spectral information is obtained from the final $D R 8$ spectroscopy files. ${ }^{51}$

\subsubsection{QSO_KNOWN}

eBOSS quasar targeting will not reobserve objects with previous good spectra (defined by the QSO_BOSS_TARGET and QSO_SDSS_TARGET bits). The purpose of the QSO_KNOWN bit is to track which previously known objects have a reliable, visually inspected (or otherwise highly confident) redshift and classification from prior spectroscopy. Objects classified as having excellent prior spectroscopy are those that are of $S D S S$ provenance and match the sample used to define known objects in BOSS (see Ross et al. 2012), or those that match the final BOSS quasar catalog (DR12Q; c.f. Pâris et al. 2014). The QSO_KNOWN bit is intended to represent the subset of objects deliberately not observed that have a reliable spectrum-because objects without such a reliable spectrum are almost certainly not quasars. The main utility of this bit is to populate catalogs for scientific analyses with reliable previous redshifts and classifications. The version of the $D R 12 Q$ catalog used to set QSO_KNOWN changed at the time of the eboss 6 tiling in the same manner as described for the QSO_BAD_BOSS bit.

\subsubsection{DO_NOT_OBSERVE: Which Previously known Quasars are Targeted?}

The parameter space for $e B O S S$ quasar targeting overlaps that of earlier iterations of the SDSS. The bits QSO_BAD_BOSS, QSO_BOSS_TARGET, QSO_SDSS_TARGET, and QSO_KNOWN work together to determine a sample of objects for which eBOSS does not need to obtain an additional spectrum because a good classification and redshift should already exist (if the object is a quasar). Targets are not observed if QSO_BOSS_TARGET, QSO_SDSS_TARGET, or QSO_KNOWN are set, unless QSO_BAD_BOSS is set. In addition, QSO_REOBS always forces a reobservation of an earlier BOSS quasar. In Boolean notation, DO_NOT_OBSERVE is then set

\footnotetext{
${ }^{50}$ Specifically the combination of v5_7_0 and v5_7_1 of the BOSS SpAll file (http://data.sdss3.org/datamodel/files/BOSS_SPECTRO_REDUX/ RUN2D/spAll.html) circa 30 May 2014.

${ }^{51}$ Specifically the (line-by-line) parallel spectroscopy and imaging catalogs at http://data.sdss3.org/sas/dr8/sdss/spectro/redux/photoPosPlate-dr8.fits and http://data.sdss3.org/sas/dr8/sdss/spectro/redux/specObj-dr8.fits
} 
according to quasar target bits if:

$$
\begin{aligned}
& \text { (QSO_KNOWN } \| \text { QSO_BOSS_TARGET } \| \\
& \text { QSO_SDSS_TARGET) } \\
& \text { and ! (QSO_BAD_BOSS } \| \text { QSO_REOBS). }
\end{aligned}
$$

The reduction in target density from implementing this schema is significant. Broadly, the total density of eBOSS CORE quasar targets that have to be allocated a fiber drops from $\sim 115 \mathrm{deg}^{-2}$ to close to $\sim 90 \mathrm{deg}^{-2}$ with effectively no loss of useful quasars (see Section 5). This filtering allows $e B O S S$ to target a larger number of Ly $\alpha$ quasars using the QSO_PTF method, and may ultimately result in a larger total area for eBOSS.

\subsubsection{DR9_CALIB TARGET: Which Version of the SDSS Imaging was Used?}

eBOSS quasar targeting always uses the updated imaging described in Section 3.1. In Section 5 we describe a preliminary survey called SEQUELS that bridged the SDSS-III and SDSS-IV surveys. SEQUELS targeted quasars selected in both the DR9 imaging used for BOSS and the updated imaging used in eBOSS. The DR9_CALIB_TARGET bit signifies quasars that were selected for SEQUELS using the DR9 imaging calibrations.

\section{RESULTS FROM A LARGE PILOT SURVEY}

The approaches discussed so far for $e B O S S$ quasar targeting were mostly based upon an $\sim 11 \mathrm{deg}^{2}$ test survey-which is further described in the appendix of Alam et al. (2015) - that was conducted in the CFHT Legacy Survey W3 field (e.g., see Sections 4.1.1 and 4.1.2). This test field alone was sufficient to define a mature eBOSS quasar targeting process, which is outlined in Section 4.4. To determine whether the targeting approaches detailed so far in this paper truly met $e B O S S$ goals and to provide a sample for initial scientific analyses, a larger pilot survey was conceived as part of SDSS-III. This section describes the targeting results from this survey, the SEQUELS, in the context of whether they meet the goals outlined in Section 2.2.

\subsection{Details of the SEQUELS Survey}

SEQUELS comprises two chunks of BOSS covering $\sim 810 \mathrm{deg}^{2}$ in total area. ${ }^{52}$ SEQUELS approximates the region bounded by the SDSS Legacy imaging footprint and $120^{\circ} \leqslant \alpha_{\mathrm{J} 2000}<210^{\circ}$ and $+45^{\circ} \leqslant \delta_{\mathrm{J} 2000}<+60^{\circ}$. Targets are selected as described thus far for $e B O S S$ with five slight differences.

1. The bright-end cut enforced on all target classes in SEQUELS was $i>17$ on FIBERMAG rather than on FIBER2MAG. This choice makes a tiny difference to the selected targets, of the order of $0.2 \%$.

2. IMAGE_STATUS flags were not applied in SEQUELS. More than $97 \%$ of the SEQUELS area has good IMAGE_STATUS according to our definition from Section 4.3. The remaining $\sim 3 \%$ of area, however, would not have been observed in $e B O S S$ proper.

\footnotetext{
$\overline{52}$ Designated boss214 and boss217; see http://www.sdss3.org/dr10/ algorithms/boss_tiling.php\#chunks for a description of BOSS chunks.
}

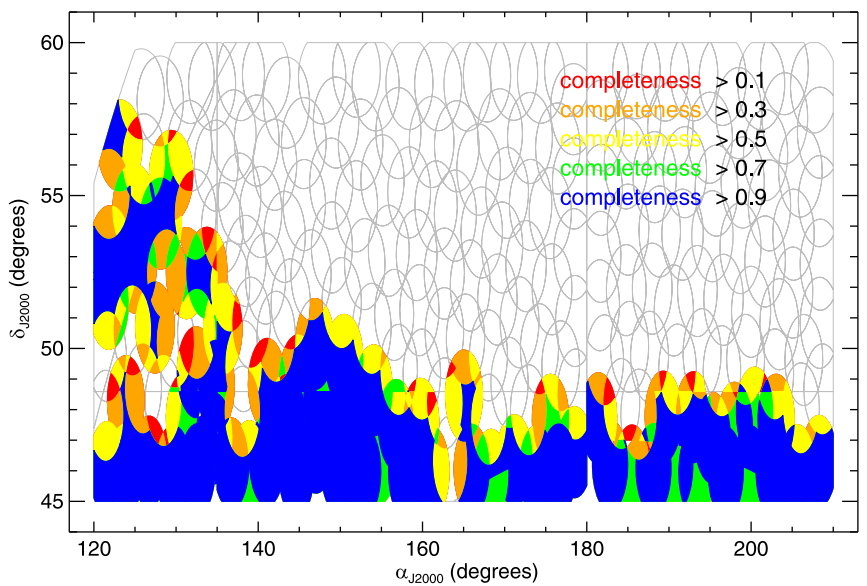

Figure 13. Targeting completeness of CORE quasars as a function of position across the first 66 plates of SEQUELS. Blue corresponds to a completeness of greater than $90 \%$, red of only greater than $10 \%$. Gray lines depict sectors of SEQUELS that have yet to be observed. The structure of the overlapping plates in defining complete areas is apparent, and the quasar density is a function of that completeness. Overall, the depicted SEQUELS plates with completenesses above zero comprise $299.3 \mathrm{deg}^{2}$ of area, but an effective area (area $\times$ targeting completeness) of only $236.3 \mathrm{deg}^{2}$.

3. The QSO_EBOSS_KDE target class (see Section 4.4) was observed in SEQUELS, but was discontinued for $e B O S S$.

4. CORE quasar targets in $e B O S S$ are all selected from the updated imaging described in Section 3.1. In SEQUELS the superset arising from both the updated and $D R 9$ imaging was targeted, because the updated imaging calibrations were considered to be preliminary. As we outline in this section, the updated imaging is sufficient to meet $e B O S S$ goals, so targeting using $D R 9$ imaging was discontinued after SEQUELS. In this section, we only discuss the results arising from the use of the updated imaging.

5. For SEQUELS the QSO_PTF target density was set at $\sim 35 \mathrm{deg}^{-2}$, which is higher than the typical $e B O S S$ density of this target class of $\sim 20 \mathrm{deg}^{-2}$.

Spectroscopic observations for SEQUELS were conducted in the same fashion as general BOSS plates (see Dawson et al. 2013), with average exposure times of 75 minutes. The SEQUELS observations contained in DR12 consist of 66 plates over an effective area of $236.3 \mathrm{deg}^{2}$. The coverage is depicted in Figure 13. The targeting completeness, defined as the fraction of all targets that have received a fiber in each overlapping sector of the survey, is plotted. ${ }^{53}$ Sectors are derived using the MANGLE software package (e.g., Swanson et al. 2008).

Every object targeted as a quasar or identified as a likely quasar by the automated pipeline (Bolton et al. 2012) was visually inspected following the procedures presented in Pâris et al. (2014). The final classifications are described in $D R 12 Q$. A summary of the results is reported in Table 3. Figures 14-16 display typical SEQUELS spectra as a function of $g$-band magnitude. It is apparent that even the faintest quasars observed in SEQUELS (Figure 16) can be identified and assigned a redshift on visual inspection, even with no smoothing or other enhancements to the spectrum. A caveat

\footnotetext{
$\overline{53}$ See Blanton et al. (2003) for the definition of a sector in the context of SDSS tiling.
} 
Table 3

Redshift and Classification Efficiency from SEQUELS for CORE Quasars upon Visual Inspection

\begin{tabular}{lcccccc}
\hline \hline $\begin{array}{l}r< \\
(1)\end{array}$ & $\begin{array}{c}f_{\text {conf }} \\
(2)\end{array}$ & $\begin{array}{c}f_{z} \\
(3)\end{array}$ & $\begin{array}{c}f_{\text {qsoconf }} \\
(4)\end{array}$ & $\begin{array}{c}f_{\text {qsoz }} \\
(5)\end{array}$ & $\begin{array}{c}f_{\text {coreconf }} \\
(6)\end{array}$ & $\begin{array}{c}f_{\text {corez }} \\
(7)\end{array}$ \\
\hline 21.0 & 0.981 & 0.960 & 0.996 & 0.970 & 0.997 & 0.973 \\
21.1 & 0.980 & 0.960 & 0.995 & 0.970 & 0.996 & 0.973 \\
21.2 & 0.978 & 0.958 & 0.994 & 0.970 & 0.996 & 0.972 \\
21.3 & 0.977 & 0.958 & 0.993 & 0.970 & 0.995 & 0.972 \\
21.4 & 0.977 & 0.957 & 0.993 & 0.970 & 0.995 & 0.972 \\
21.5 & 0.975 & 0.956 & 0.992 & 0.969 & 0.995 & 0.972 \\
21.6 & 0.971 & 0.953 & 0.991 & 0.968 & 0.993 & 0.971 \\
21.7 & 0.968 & 0.950 & 0.989 & 0.967 & 0.992 & 0.970 \\
21.8 & 0.964 & 0.947 & 0.987 & 0.966 & 0.990 & 0.970 \\
21.9 & 0.960 & 0.944 & 0.986 & 0.966 & 0.989 & 0.969 \\
22.0 & 0.957 & 0.941 & 0.984 & 0.965 & 0.987 & 0.968 \\
\hline
\end{tabular}

Note. (1) The $r$ limit for which the efficiencies are derived; (2) The fraction of all quasar targets with a highly confident classification and redshift; (3) The fraction of all quasar targets for which the SDSS spectroscopic pipeline redshift is accurate; (4)-(5) As for columns (2)-(3) but for targets classified as quasars on visual inspection; (6)-(7) As for columns (2)-(3) but for quasar targets classified as $0.9<z<2.2$ (i.e., "CORE") quasars on visual inspection.

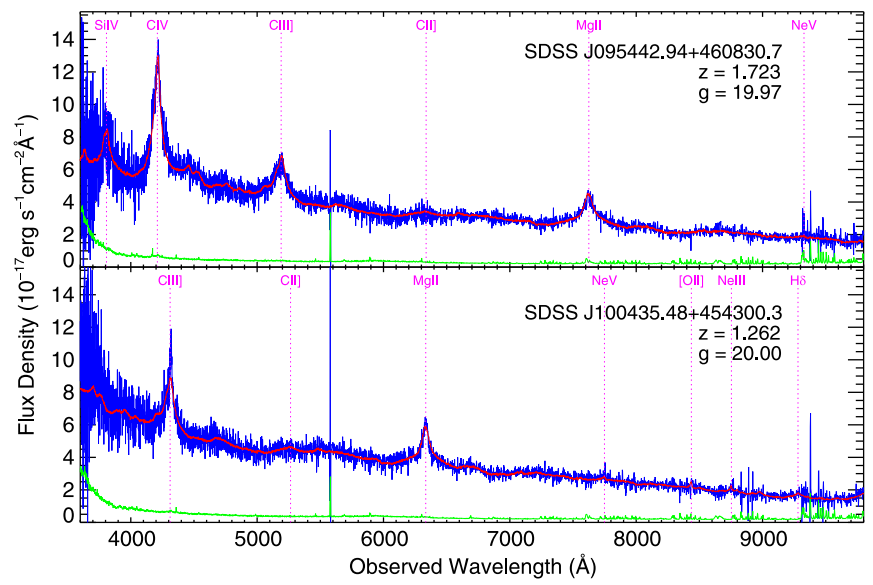

Figure 14. Two representative spectra of $g \sim 20$ quasars from SDSS plate 7284 (part of SEQUELS). Plate 7284 had a total exposure time of 75 minutes. The spectra have not been smoothed or otherwise enhanced. The dotted lines and associated labels mark the positions of some typical quasar emission lines with restframe wavelengths taken from Vanden Berk et al. (2001). Emission lines that are close to the edges of the covered wavelength range are not marked. Other labels are the object name, redshift, and (observed, not deextincted) $g$-band target magnitude. The blue solid line depicts the flux density $\left(f_{\lambda}\right)$, the green depicts the $1 \sigma$ error on $f_{\lambda}$, and the red depicts the best-fit template output by the SDSS pipeline.

is that SEQUELS was conducted during particularly good observing conditions, and there is therefore no guarantee that the quality of SEQUELS spectra will be representative of the full $e B O S S$ survey.

Based on Table 3, we expect that of the order of $96 \%$ of all quasar targets in eBOSS will be confidently classified to $r<22$, and $\simeq 99 \%$ of CORE quasars should be confidently identified. There are a number of reasons to believe that SEQUELS may slightly overestimate our ability to classify quasars in every area of the $e B O S S$ survey. First, the SEQUELS area contains relatively good imaging when compared to several $e B O S S$ areas in the SDSS SGC region (see Section 6). Second, as SEQUELS occurred concurrently with $B O S S$ observations, some $z>2$ BOSS quasars that would not

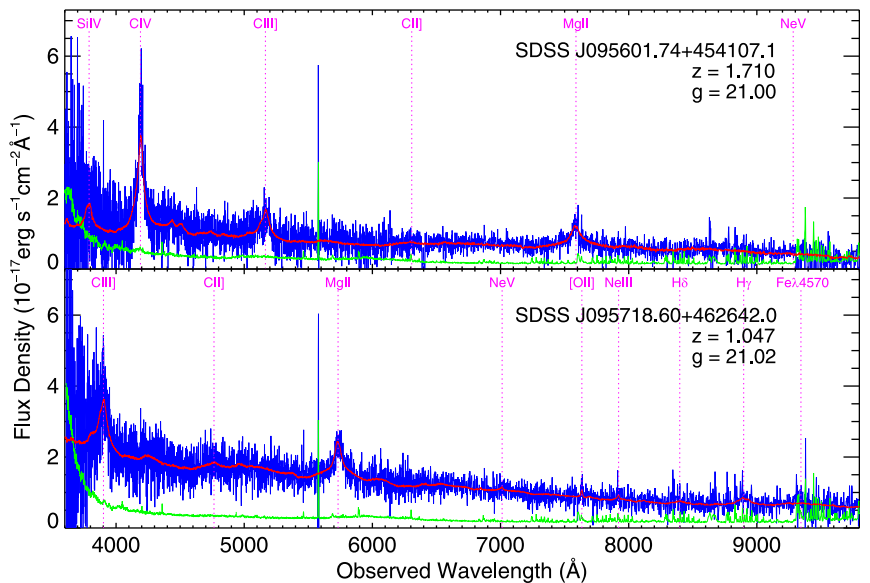

Figure 15. As for Figure 14 but for $g \sim 21$ quasars.

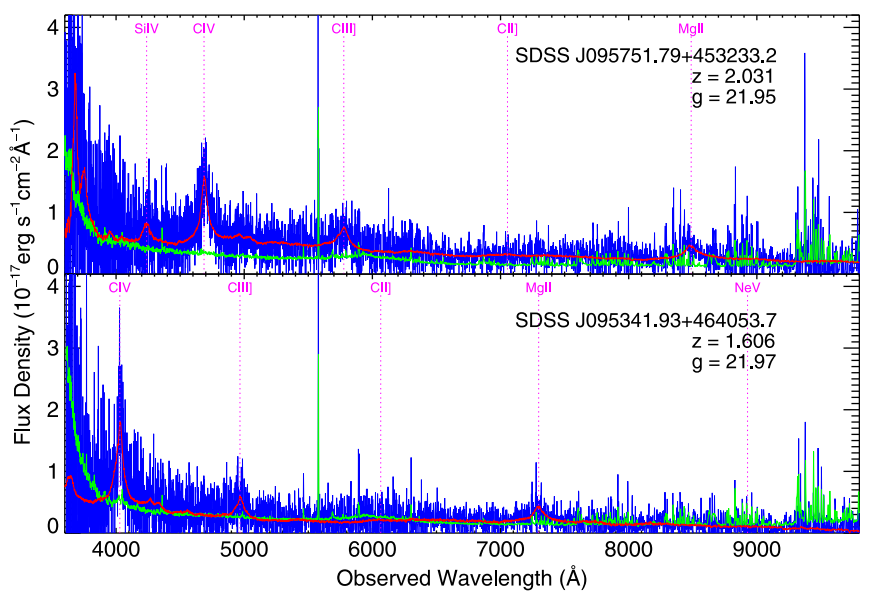

Figure 16. As for Figure 14, but for $g \sim 22$ quasars.

be reobserved in eBOSS were tagged as SEQUELS targetsand, in general, $z>2$ quasars are easier to classify as the strong $\operatorname{Ly} \alpha$ line and the Ly $\alpha$ Forest are redshifted into the $B O S S$ spectrograph bandpass at about $z>2$. More comprehensive details of the $e B O S S$ pipeline and spectral classification procedures - and, in particular, whether the pipeline meets the requirements discussed in Section 2.2-are provided in our companion overview paper (Dawson et al. 2015).

\subsection{Projected eBOSS Targeting Efficiency}

Perhaps the most critical aspect of $e B O S S$ quasar targeting is that a sufficiently high density of quasars is obtained to make meaningful and/or improved measurements of the $\mathrm{BAO}$ distance scale. Contingent on the effective area of SEQUELS (as depicted in Figure 13), we can estimate the quasar density expected for $e B O S S$. Making this estimate is relatively straightforward: it is obtained by dividing the total number of spectroscopically confirmed quasars in SEQUELS by the completeness-weighted area of the survey as a function of targeting approach and redshift. For this purpose, "completeness" means targeting completeness to the statistically selected quasar sample, which here is defined as the fraction of CORE quasar targets that received a fiber for spectroscopic observation. Targeting incompleteness occurs in SEQUELS for two main reasons: First, due to collisions, a fiber cannot always be placed on neighboring targets, causing 
Table 4

Density of SEQUELS Quasar Targets that are Confidently a Quasar upon Visual Inspection

\begin{tabular}{|c|c|c|c|c|c|c|c|c|c|c|c|}
\hline \multirow{2}{*}{$\begin{array}{l}\text { Comp. } \\
> \\
\text { (1) }\end{array}$} & \multirow{2}{*}{$\begin{array}{c}\text { Total } \\
\text { Area } \\
(2)\end{array}$} & \multirow{2}{*}{$\begin{array}{l}\text { Eff. } \\
\text { Area } \\
(3) \\
\end{array}$} & \multicolumn{3}{|c|}{$0.9<z<2.2$ from CORE } & \multicolumn{3}{|c|}{ ALL $z$ from CORE } & \multicolumn{3}{|c|}{ New $z>2.1$ from... } \\
\hline & & & $\begin{array}{c}\text { New } \\
\text { (4) }\end{array}$ & $\begin{array}{c}\text { Known } \\
\text { (5) }\end{array}$ & $\begin{array}{c}\text { Total } \\
\text { (6) }\end{array}$ & $\begin{array}{c}\text { New } \\
\text { (7) }\end{array}$ & $\begin{array}{c}\text { Known } \\
(8)\end{array}$ & $\begin{array}{c}\text { Total } \\
\text { (9) }\end{array}$ & $\begin{array}{c}\text { CORE } \\
(10)\end{array}$ & $\begin{array}{l}\text { PTF } \\
(11)\end{array}$ & $\begin{array}{c}\text { Total } \\
\text { (12) }\end{array}$ \\
\hline 0.80 & 189.9 & 183.5 & 58.3 & 13.4 & 71.6 & 69.7 & 29.0 & 98.7 & 6.6 & 4.6 & 11.2 \\
\hline 0.85 & 187.6 & 181.6 & 58.3 & 13.3 & 71.6 & 69.7 & 29.0 & 98.7 & 6.6 & 4.4 & 11.0 \\
\hline 0.90 & 174.5 & 170.0 & 58.4 & 13.4 & 71.8 & 69.8 & 29.2 & 99.0 & 6.6 & 4.4 & 11.0 \\
\hline 0.95 & 125.9 & 124.7 & 59.2 & 12.8 & 72.0 & 71.0 & 27.9 & 98.9 & 7.0 & 4.1 & 11.1 \\
\hline
\end{tabular}

Note. (1) Targeting completeness (fraction of CORE targets that received a fiber) limit of the sectors used for a given row of the table (see also Figure 13). eBOSS should be $>95 \%$ complete; (2) Total SEQUELS area above this completeness ( deg $^{2}$ ); (3) The effective area (area in deg ${ }^{2}$ weighted by per-sector completeness); (4) Completeness-weighted total density of new (i.e., previously unconfirmed) $0.9<z<2.2$ quasars $\left(\mathrm{deg}^{-2}\right)$ targeted by the CORE (i.e., having the QSO_EBOSS_CORE bit set). We define a quasar as an object classified QSO or QSO_Z? as in Table 2 of Pâris et al. (2014); (5) The total density of previously confirmed $0.9<z<2.2$ quasars from earlier SDSS surveys $\left(\mathrm{deg}^{-2}\right.$ ) targeted by the CORE; (6) Total density (completeness-weighted) of $0.9<z<2.2$ quasars that would comprise the CORE clustering sample $\left(\mathrm{deg}^{-2}\right)$. We only include objects classified as a quasar-a further $1.5-2 \mathrm{deg}^{-2}$ of CORE targets are galaxies (or unidentifiable objects) at $0.9<z<2.2$; (7)-(9) As for columns (4)-(6) but for all quasars selected by the CORE (not just those that are at $0.9<z<2.2$ on visual inspection); (10) New quasars selected by the CORE as for columns (4) and (7) but specifically at $z>2.1$ (the Ly $\alpha$ quasar redshift range); (11) New quasars (heterogeneously) selected by only PTF (i.e., having the QSO_PTF bit set), this column is not completeness-weighted; (12) Total density of new z $>2.1$ quasars that would comprise the $e$ BOSS sample of Ly $\alpha$ quasars.

general incompleteness on a plate; and, second, certain plates in SEQUELS are yet to be observed, causing significant incompleteness in areas where yet-to-be-observed plates overlap completed plates. Table 4 presents estimates of the $e B O S S$ quasar density. In addition to weighting the CORE quasar counts by completeness on a sector-by-sector basis, Table 4 details results as a function of completeness. Ultimately, eBOSS is expected to have a targeting completeness of 0.95 (due to collisions, fibers will only be placed on $95 \%$ of quasar targets), so it is worth noting that the statistics in Table 4 are somewhat dependent on completeness.

The results in Table 4 have been produced in a manner that should reflect the eventual targeting schema for eBOSS. One subtlety is that most, but not all, BOSS observations had been completed in the depicted area in Figure 13 by the time of SEQUELS observations. To better mimic eBOSS, estimates in Table 4 are produced by substituting non-SEQUELS (BOSS) identifications from $D R 12 Q$ over SEQUELS targets, where they exist, and such objects are treated as previously observed, known quasars (i.e., when such objects have a good spectrum from $D R 12 Q$, they are treated as if they had a known redshift from BOSS and as if the DO_NOT_OBSERVE bit had been set; see Section 4.4.10). At the outset of SEQUELS, 8921 potential SEQUELS targets had the DO_NOT_OBSERVE bit set due to a prior good spectrum in SDSS-I, II, or III. Based on our substitution process, only an additional $267(\sim 3 \%)$ quasars would have had the DO_NOT_OBSERVE bit set due to yet-tobe-completed BOSS observations, and only $92(\sim 1 \%)$ of these additional quasars would have been in the redshift range $0.9<z<2.2$.

It is critical for users of $e B O S S$ data to be able to accurately track previously known quasars from earlier versions of the SDSS. Table 4 implies that of the order of $\sim 13 \mathrm{deg}^{-2}$ $0.9<z<2.2$ quasars will be included in $e B O S S$ as a prior confirmation. This number of $\sim 13 \mathrm{deg}^{-2}$ previously identified CORE quasars is as might be expected. The SDSSI/II quasar catalog of Schneider et al. (2010) contains $\sim 75,000$ $0.9<z<2.2$ quasars spread over $9400 \mathrm{deg}^{2} \quad\left(\sim 8 \mathrm{deg}^{-2}\right)$. The BOSS quasar catalog of $D R 12 Q$ contains $\sim 65,000$ $0.9<z<2.2$ quasars spread over $10,700 \mathrm{deg}^{2}\left(\sim 6 \mathrm{deg}^{-2}\right)$. These catalogs also contain $\sim 1 \mathrm{deg}^{-2}$ mutual $0.9<z<2.2$ quasars. Depending on SEQUELS sector, the number of known quasars in the CORE redshift range can vary widely, from as few as $5 \mathrm{deg}^{-2}$ to as many as $25 \mathrm{deg}^{-2}$ due to the complex set of ancillary programs that were conducted as part of BOSS (see, e.g., Dawson et al. 2013).

The main purpose of this section is to investigate whether the eBOSS target selection as applied to SEQUELS meets the requirements discussed in Section 2.2, which amounts to a success rate of $>58 \mathrm{deg}^{-2} 0.9<z<2.2$ quasars over $7500 \mathrm{deg}^{2}$. Whether the area requirements of Section 2.2 will be met are discussed in Dawson et al. (2015). The results from the SEQUELS area suggest that $e B O S S$ will meet its quasar targeting requirements in terms of number densities. For a targeting completeness reflective of eBOSS ( $\sim 95 \%)$, a completeness-weighted density of $72.0 \mathrm{deg}^{-2} 0.9<z<2.2$ quasars were identified in SEQUELS. This suggests that the eBOSS CORE quasar selection will identify $(0.95 \times 72.0=)$ $68.4 \mathrm{deg}^{-2} 0.9<z<2.2$ quasars.

The SDSS imaging in the SEQUELS area may be of aboveaverage quality, which could inflate these expectations (see Section 6). There are also reasons to believe, however, that the eBOSS quasar density may be higher than SEQUELS expectations. For instance, SEQUELS data were reduced using the SDSS-III spectroscopic pipeline, which, with augmentations, might improve on the $\sim 1 \%$ loss due to unidentifiable quasars listed in Table 3. Also, there are $1.5-2 \mathrm{deg}^{-2}$ additional objects in the CORE redshift range in SEQUELS that are not included in Table 4 because they are classified as "unknown" or as galaxies upon visual inspection. In theory, these objects can also be used for $e B O S S$ clustering analyses (although such objects have a median redshift of $\sim 1.1$ ).

Fibers not allocated to other eBOSS target classes are assigned to finding new Ly $\alpha$ quasars $(z>2.1)$. In Table 4 we show that SEQUELS contains $(7.0 \times 0.95)=\sim 6.7 \mathrm{deg}^{-2}$ new Ly $\alpha$ quasars acquired by the CORE selection and $(4.1 \times 0.95)=\sim 3.9 \mathrm{deg}^{-2}$ new Ly $\alpha$ quasars acquired by other selections (mainly objects with the QSO_PTF bit set). These results are likely robust for CORE targets (given the caveats discussed in the previous paragraph). Ly $\alpha$ quasar target density may fluctuate across the survey with the availability of $P T F$ imaging (see Section 4.2.1), so SEQUELS is a reasonable 


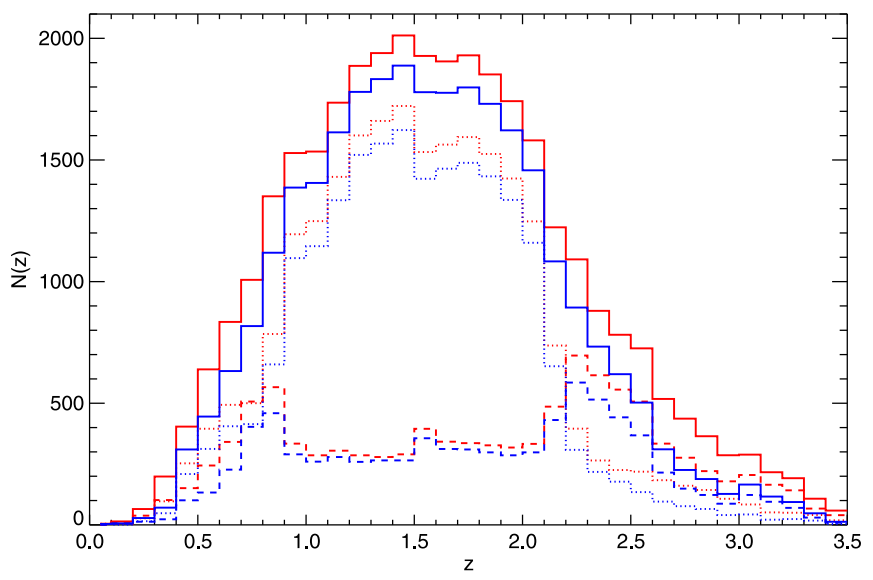

Figure 17. Redshift distribution of quasars from SEQUELS. Red lines represent all quasars identified in SEQUELS, blue lines represent quasars targeted just by the CORE algorithm, and solid lines represent all quasars that would have been assigned a fiber by the SEQUELS targeting algorithm (i.e., including known SDSS or BOSS quasars that do not need to be reobserved because they have the DO_NOT_OBSERVE bit set). Dashed (dotted) lines represent quasars that were (were not) previously spectroscopically confirmed in the SDSS or BOSS. The solid lines, which are the sum of the dotted and dashed lines, are quantified in columns 3 and 6 of Table 5 and have been completeness-corrected as described in that table.

but imperfect estimate of the success rate for new QSO_PTF Ly $\alpha$ quasars in $e B O S S$. In particular, the target density of QSO_PTF sources was $35 \mathrm{deg}^{-2}$ in SEQUELS, but is expected to be close to $20 \mathrm{deg}^{-2}$ across the entire $e B O S S$ footprint (see Section 5.1). Thus expected density of new $z>2.1$ quasars from the $e B O S S Q S O \_P T F$ program is quoted as $3-4 \mathrm{deg}^{-2}$ in the abstract of this paper. There are also reasons to believe, however, that results from SEQUELS may underestimate the success of $e B O S S$. Most notably, our companion surveys such as TDSS (Morganson et al. 2015) target some Ly $\alpha$ quasars in addition to those targeted by the QSO_EBOSS_CORE and QSO_PTF approaches (see, e.g., J. Ruan et al. 2016, in preparation).

\subsection{Overall Characteristics of eBOSS Quasars}

Beyond the cosmological goals of $e B O S S$, the quasar sample produced by $S D S S-I V$ should be unparalleled, exceeding the depth and numbers of any previous quasar sample. As there is likely to be significant interest in the nature of eBOSS for quasar science, quasars observed as part of SEQUELS are broadly characterized in this section. Because SEQUELS observations were conducted in tandem with BOSS, some quasars that would not normally receive a fiber in eBOSS because of existing BOSS spectroscopy did receive a SEQUELS fiber. Throughout this section, we treat such objects as if they had the DO_NOT_OBSERVE bit set by correctly incorporating (non-SEQUELS) redshifts and classifications from the DR12 quasar catalog (I. Pâris et al. 2016, in preparation), as also described in the discussion of Table 4 in Section 5.2.

The redshift distribution of quasars in SEQUELS is plotted in Figure 17 and is similar to the expectation from Figure 4 . The measurements of the SEQUELS $N(z)$ are listed in Table 5. When combined with the expected total $e B O S S$ quasar target density over all redshifts of $\sim 99 \mathrm{deg}^{-2}$ (see Table 4) and the expected $7500 \mathrm{deg}^{2}$ area of $e B O S S$, the SEQUELS N(z) should be sufficient to project science results using an $e B O S S$-like
Table 5

$N(z)$ for $S E Q U E L S$ Quasars upon Visual Inspection

\begin{tabular}{|c|c|c|c|c|c|c|}
\hline \multirow[b]{2}{*}{$\begin{array}{l}z \\
\text { (1) }\end{array}$} & \multicolumn{3}{|c|}{ CORE Quasars } & \multicolumn{3}{|c|}{ All Quasars } \\
\hline & $\begin{array}{r}N_{\text {raw }} \\
\text { (2) }\end{array}$ & $\begin{array}{l}N \\
\text { (3) }\end{array}$ & $\begin{array}{l}d N \\
\text { (4) }\end{array}$ & $\begin{array}{r}N_{\text {raw }} \\
\text { (5) }\end{array}$ & $\begin{array}{l}N \\
(6)\end{array}$ & $\begin{array}{l}d N \\
\text { (7) }\end{array}$ \\
\hline 0.05 & 3 & 3.8 & 0.001 & 4 & 4.8 & 0.001 \\
\hline 0.15 & 6 & 6.3 & 0.002 & 14 & 14.3 & 0.004 \\
\hline 0.25 & 25 & 28.1 & 0.010 & 62 & 65.1 & 0.019 \\
\hline 0.35 & 61 & 70.8 & 0.025 & 189 & 198.8 & 0.059 \\
\hline 0.45 & 267 & 310.0 & 0.108 & 361 & 404.0 & 0.120 \\
\hline 0.55 & 381 & 445.2 & 0.155 & 575 & 639.2 & 0.190 \\
\hline 0.65 & 549 & 632.4 & 0.221 & 751 & 834.4 & 0.249 \\
\hline 0.75 & 732 & 817.2 & 0.285 & 922 & 1007.2 & 0.300 \\
\hline 0.85 & 983 & 1118.7 & 0.390 & 1215 & 1350.7 & 0.402 \\
\hline 0.95 & 1161 & 1386.6 & 0.484 & 1303 & 1528.6 & 0.455 \\
\hline 1.05 & 1170 & 1405.7 & 0.490 & 1299 & 1534.7 & 0.457 \\
\hline 1.15 & 1339 & 1613.5 & 0.563 & 1461 & 1735.5 & 0.517 \\
\hline 1.25 & 1467 & 1779.9 & 0.621 & 1574 & 1886.9 & 0.562 \\
\hline 1.35 & 1510 & 1832.5 & 0.639 & 1617 & 1939.5 & 0.578 \\
\hline 1.45 & 1555 & 1887.9 & 0.659 & 1679 & 2011.9 & 0.599 \\
\hline 1.55 & 1485 & 1778.7 & 0.620 & 1634 & 1927.7 & 0.574 \\
\hline 1.65 & 1475 & 1776.2 & 0.620 & 1604 & 1905.2 & 0.568 \\
\hline 1.75 & 1493 & 1798.1 & 0.627 & 1625 & 1930.1 & 0.575 \\
\hline 1.85 & 1435 & 1730.7 & 0.604 & 1556 & 1851.7 & 0.552 \\
\hline 1.95 & 1347 & 1621.8 & 0.566 & 1467 & 1741.8 & 0.519 \\
\hline 2.05 & 1219 & 1457.5 & 0.508 & 1342 & 1580.5 & 0.471 \\
\hline 2.15 & 949 & 1083.2 & 0.378 & 1089 & 1223.2 & 0.364 \\
\hline 2.25 & 833 & 893.5 & 0.312 & 1031 & 1091.5 & 0.325 \\
\hline 2.35 & 685 & 732.8 & 0.256 & 832 & 879.8 & 0.262 \\
\hline 2.45 & 584 & 619.5 & 0.216 & 746 & 781.5 & 0.233 \\
\hline 2.55 & 474 & 502.7 & 0.175 & 697 & 725.7 & 0.216 \\
\hline 2.65 & 291 & 310.7 & 0.108 & 498 & 517.7 & 0.154 \\
\hline 2.75 & 211 & 225.8 & 0.079 & 423 & 436.8 & 0.130 \\
\hline 2.85 & 174 & 188.5 & 0.066 & 349 & 364.5 & 0.109 \\
\hline 2.95 & 120 & 127.3 & 0.044 & 280 & 286.3 & 0.085 \\
\hline 3.05 & 156 & 165.8 & 0.058 & 278 & 288.8 & 0.086 \\
\hline 3.15 & 112 & 116.4 & 0.041 & 212 & 216.4 & 0.064 \\
\hline 3.25 & 89 & 93.9 & 0.033 & 188 & 191.9 & 0.057 \\
\hline 3.35 & 44 & 47.6 & 0.017 & 103 & 107.6 & 0.032 \\
\hline 3.45 & 12 & 12.8 & 0.004 & 58 & 58.8 & 0.018 \\
\hline 3.55 & 9 & 10.8 & 0.004 & 58 & 59.8 & 0.018 \\
\hline 3.65 & 6 & 7.0 & 0.002 & 61 & 62.0 & 0.018 \\
\hline 3.75 & 8 & 9.6 & 0.003 & 51 & 50.6 & 0.015 \\
\hline 3.85 & 6 & 6.5 & 0.002 & 37 & 39.5 & 0.012 \\
\hline 3.95 & 4 & 4.5 & 0.002 & 27 & 27.5 & 0.008 \\
\hline 4.05 & 3 & 3.3 & 0.001 & 19 & 19.3 & 0.006 \\
\hline 4.15 & 2 & 2.5 & 0.001 & 10 & 10.5 & 0.003 \\
\hline
\end{tabular}

Note. (1) Redshift; (2) Number of SEQUELS quasars selected by the CORE targeting algorithm; (3) As for column (2) but completeness-corrected; (4) As for column (3) but normalized; (5)-(7) As for columns (2)-(4) but for SEQUELS quasars selected by any targeting algorithm. Completeness corrections are conducted by multiplying the counts of all newly identified CORE quasars by 298.5/237.1 (see the first row of Table 4). Counts of all other quasars in SEQUELS are not completeness-corrected because they are dominated by quasars that were previously confirmed in the SDSS or BOSSsuch quasars are effectively assigned a fiber $100 \%$ of the time. A quasar is defined using QSO or QSO_Z? as in Table 4.

sample. The redshift-absolute-magnitude distribution of SEQUELS is provided in Figure 18. This figure illustrates why $e B O S S$ will be the next-generation quasar survey, complementing the (largely) $i<19$ space of $S D S S-I / I I$ and 


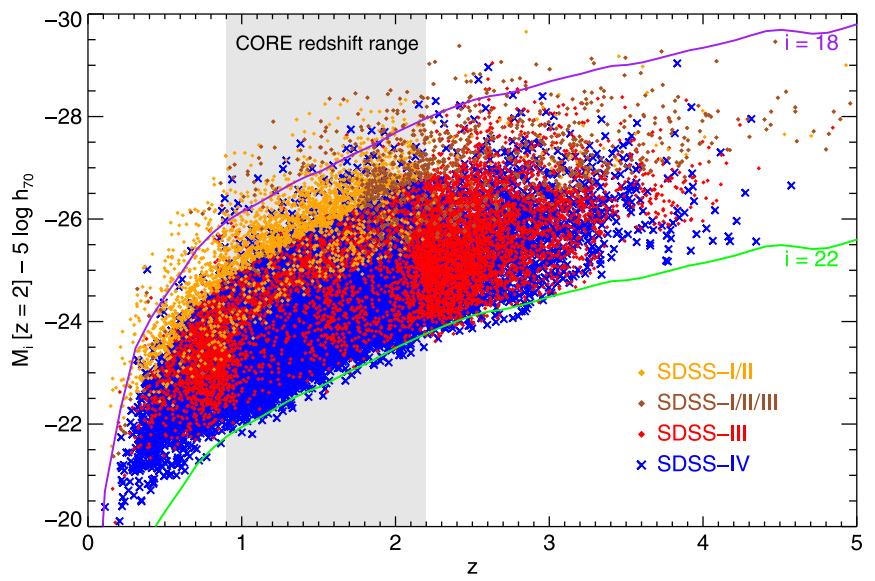

Figure 18. The ( $i$-band) absolute-magnitude-redshift plane for quasars targeted in SEQUELS. The blue crosses depict new quasars that would be observed as part of $S D S S-I V / e B O S S$. The other points represent quasars that would be targeted by $e B O S S$ but would not receive a fiber due to being previously observed in SDSS-I/II (orange), SDSS-III (red), or in both (brown; mostly ancillary targets or QSO_KNOWN_SUPPZ targets; see Dawson et al. 2013). The lines track quasars representative of the extremes of SDSS target selection between $i=18$ (purple) and $i=22$ (green). The gray box illustrates the power of eBOSS for detecting new quasars in the CORE redshift range. All magnitudes are based on PSF fluxes and have been de-extincted. Absolute magnitudes have been $K$-corrected to $z=2$ using Table 4 of Richards et al. (2006) and assume $H_{0}=70 \mathrm{~km} \mathrm{~s}^{-1} \mathrm{Mpc}^{-1}$.

the (largely) $z<0.9$ and $z>2.2$ space of $B O S S$, by filling in the $i>19$ and $0.9<z<2.2$ quasar space in an unprecedented fashion.

The overall expected quasar numbers for eBOSS can be estimated from the SEQUELS $N(z)$ and number densities. Projecting from Table 4 and assuming a minimum $e B O S S$ area of $7500 \mathrm{deg}^{2}$ (Section 2.2), eBOSS should, conservatively, comprise at least 500,000 spectroscopically confirmed $0.9<z<2.2$ quasars selected in a uniform manner with which to pursue quasar clustering studies such as the BAO scale, and at least 500,000 total new quasars (at any redshift) that have never been spectroscopically identified and characterized. Overall, at the completion of eBOSS, the SDSS surveys will have provided unique spectra of more than 800,000 total quasars, including SDSS areas outside the $e B O S S$ footprint and new quasars observed by the TDSS and SPIDERS surveys.

\section{TESTS OF THE HOMOGENEITY OF THE CORE QUASAR SAMPLE}

In order to perform clustering measurements to characterize the BAO scale, it is necessary to mimic the angular distribution imposed by the target selection. This survey "mask" is often expressed as a random catalog, or control sample, that mimics the characteristics of the targeted population but in the absence of any clustering. At its simplest, this process involves uniformly distributing random points over the footprint of the target imaging. This simple approach, however, is rarely adequate because survey systematics such as seeing, sky brightness, and Galactic extinction alter the target density in a complex manner. A related issue is that zero-point calibrations in $S D S S$ imaging can vary across the survey, also producing non-cosmological variations in target density.

\subsection{Target Density Fluctuations Due to Systematics}

Previous studies of large-scale galaxy clustering over the SDSS footprint (e.g., Ross et al. 2011) have demonstrated that systematics that produce target density variations at a level of $\sim 15 \%$ or less can be controlled for by weighting the random catalog by a model of the effect of that systematic. Beyond the $15 \%$ level, systematics become more difficult to "weight" for, perhaps because some major systematics are covariant. When the effect of systematics exceeds the $15 \%$ level, that area of the survey may have to be excised from clustering analyses.

As part of eBOSS target selection, a set of regression tests were devised to study how possible systematics in SDSS and WISE imaging may affect target density-and whether such effects are below the $\sim 15 \%$ level that could be modeled with a suitable weighting scheme. The slate of systematics, which represents a reasonable (but not necessarily exhaustive) list of quantities that could bias eBOSS target density, is further detailed in a companion paper (Prakash et al. 2015b). Relevant to the WISE imaging; the systematics include the median numbers of exposures per pixel, the fraction of exposures contaminated by the moon, and the total flux per pixel, all in the W1 band (W1covmedian, moon_lev, W1median). Relevant to the SDSS imaging, the systematics include the FWHM and background sky-level in SDSS z-band, which are used to track the quality of the seeing and the sky brightness. Additional systematics include Galactic latitude (to map the density of possible contaminating stars) and Galactic dust (extinction in the $r$-band is used to represent this systematic).

The adopted regression technique is also detailed in Prakash et al. (2015b). Briefly, the potential eBOSS imaging footprint is deconstructed into equal-area pixels of $0.36 \mathrm{deg}^{2}$. The $e B O S S$ CORE quasar target density and the mean value of each systematic is determined for each of these pixels. The observed surface density $\left(\mathrm{SD}_{\mathrm{obs}}\right)$ of $e B O S S \mathrm{CORE}$ quasar targets in each pixel can be expressed as a linear model of systematics

$$
\mathrm{SD}_{\mathrm{obs}}=S_{0}+\sum_{i=1}^{7} S_{i} x_{i}+\epsilon,
$$

where $S_{0}$ is the mean target density across the pixels, $S_{i}$ is the weight accorded to fluctuations in target density $\left(x_{i}\right)$ due to systematic $i$, and $\epsilon$ is the combined effect of noise and variance, which is approximated as a Gaussian. Multilinear regression is used to determine $S_{0}$ and $S_{i}$ by minimizing the value of reduced $\chi^{2}$ across the pixels. This regression is conducted separately in each Galactic hemisphere, such that different coefficients are derived for the NGC and SGC regions of the SDSS imaging.

Once the coefficients of the linear regression model for systematics have been established, a statistic designated the Predicted Surface Density, PSD, is computed. The PSD is obtained by using $S_{0}$ and $S_{i}$ to calculate what the $e B O S S$ CORE quasar density should be in a given pixel if the linear regression model is an adequate description

$$
\mathrm{PSD}=S_{0}+\sum_{i=1}^{7} S_{i} x_{i}
$$

Figure 19 presents a histogram of the CORE quasar PSD as predicted from the derived linear regression model coefficients across all of the systematics. A total of $96.7 \%$ of the SDSS imaging footprint in the NGC fluctuates in CORE quasar PSD 


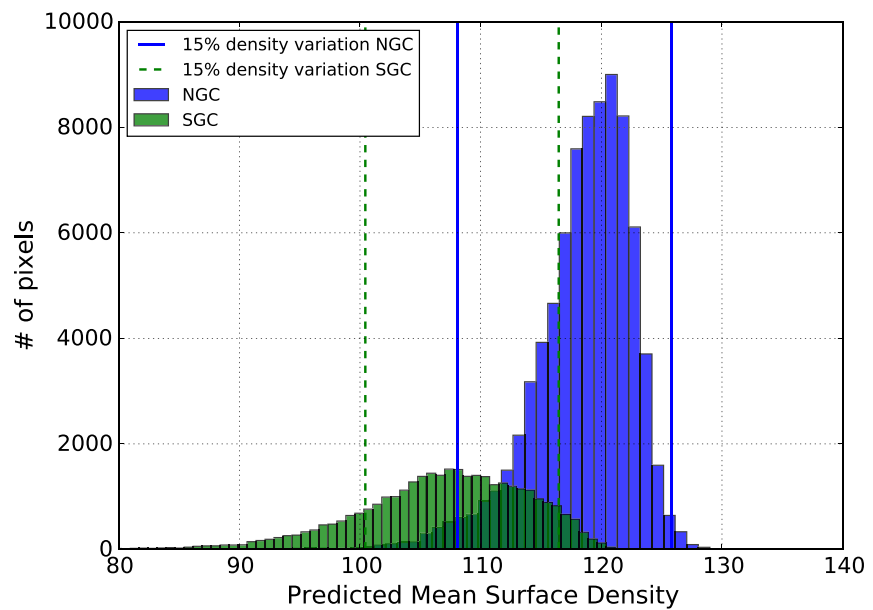

Figure 19. Histograms of the surface density of CORE quasar targets predicted by the regression models described in Section 6.1 (the PSD). The blue histogram represents the NGC, with solid blue lines depicting the window within which angular fluctuations in quasar target density meet the $\leqslant 15 \%$ requirement of Section 2.2. The green histogram and dotted green lines depict the same quantities for the SGC. The histograms demonstrate that $\sim 97 \%$ ( $\sim 77 \%$ ) of the NGC (SGC) footprint meets the homogeneity requirements of $e B O S S$ (see Section 2.2). The PSD and the fractional deviation from the mean PSD in each pixel are depicted as a sky map in Figure 20.

at less than $15 \% .^{54}$ The corresponding fraction is $76.7 \%$ in the SGC footprint.

Figure 20 illustrates these deviations on the sky using a map of the PSD statistic, which serves to illustrate the most problematic areas of the SDSS footprint for $e B O S S$. The righthand panel of Figure 20 approximates the "mask" that will be necessary to ameliorate the effects of systematics on clustering measurements that use $e B O S S$ CORE quasars. The effective area or random catalog in each region of the $e B O S S$ footprint can be re-weighted by the values displayed in the right-hand panel of Figure 20, although regions that deviate by more than $15 \%$ from expectation may need to be excised from the survey in order to reach the target density variation requirement of Section 2.2. The central panel of Figure 20 is a particularly clear illustration of why the PSD is regressed separately in the NGC and SGC regions- the NGC appears to be more robust to systematics than the SGC.

To determine whether a linear regression adequately models the effect of systematics on the target density of $e B O S S$ CORE quasars, calculate the statistics designated the Reduced_PSD ${ }_{j}$ and the Residual_PSD ${ }_{j}$ in Prakash et al. (2015b). The Reduced_PSD ${ }_{j}$ is derived from the PSD by omitting the $j$ 'th systematic term when calculating the PSD, in order to represent the deviation from the PSD caused by each systematic. The difference between the PSD and the observed sky density of targets, called the Residual Surface Density, Residual_SD, is then calculated. If a linear model is an appropriate representation of the regression of a given systematic, then the Residual_PSD should be well-represented by a model with a slope of $S_{j}$. Formally,

$$
\begin{aligned}
& \text { Reduced_PSD }=\mathrm{PSD}-S_{j} \times x_{j}, \\
& \text { Residual_SSD }=S D_{\mathrm{obs}}-\text { Reduced_PSD } \\
&
\end{aligned} .
$$

\footnotetext{
54 Only the area that could be useful for $e B O S S$ targeting, due to scheduling constraints, is considered (see Dawson et al. 2015, and Figure 20).
}

Figure 21 shows how the CORE quasar Residual_SD varies as a function of each of the individual systematics, together with the underlying distributions of those systematics. In general, a linear regression seems to be adequate for modeling variations in CORE quasar target density. Figure 21 suggests that sky brightness, and, in particular, Galactic extinction, are the main culprits in causing variations in $e B O S S$ CORE quasar target density. The SGC has a $68 \%$ range of $r$-band extinction of 0.075 to 0.19 with a median of 0.12 , whereas the NGC has a $68 \%$ range of $r$-band extinction of 0.032 to 0.10 , with a median of only 0.057 . The corresponding numbers for $z$-band sky flux are 4.1-6.8 with a median of 5.1 in the SGC and 3.3-4.6 with a median of 3.8 in the NGC. The higher median and wider range of values of these systematics in the SGC are likely responsible for both the suppressed density of SGC targets and the larger rms in predicted surface density that can be seen in Figure 20. These systematics will act to reduce the effective depth of an exposure and hence increase the error on the fluxes of a test object being assigned a quasar probability by the $\mathrm{XDQSOz}$ method. In effect, as the flux errors for a test object increase, the formal probability that the object is a quasar is reduced, and fewer objects are then assigned $\operatorname{PQSO}(z>0.9)>0.2$ by $\mathrm{XDQSOz}$.

Overall, the eBOSS quasar targeting algorithm outlined in this paper is expected to produce quasar samples for clustering measurements that are robust against systematics across essentially the entire NGC and across about three-quarters of the SGC. This statement may be pessimistic, as $e B O S S$ does not attempt to restrict the CORE quasar redshift range to $0.9<z<2.2$ in advance of spectroscopic confirmation. Quasars at $z>2.2$ are closer to the stellar locus in optical color space, so the target density of quasars at $z>2.2$ may fluctuate more due to systematics than at $z<2.2$. Weighting for systematics as a function of quasar redshift is a possible avenue for further improving $e B O S S$ clustering measurements once target redshifts have been confirmed by spectroscopy. The final $e B O S S$ footprint is yet to be derived, but in a worst-case scenario if the entire SGC has to be observed, only $\sim 86.7 \%$ of $e B O S S$ will meet the requirements of Section 2.2. This fraction of useful area is almost exactly offset by the expected excess of eBOSS CORE quasars. Table 4 implies that eBOSS will confirm $(0.95 \times 72.0=) 68.4 \mathrm{deg}^{-2} 0.9<z<2.2$ quasars. Serendipitously, $68.4 \mathrm{deg}^{-2} \times 0.867=59.3 \mathrm{deg}^{-2}$, exceeding the requirement of $58 \mathrm{deg}^{-2} 0.9<z<2.2$ quasars noted in Section 2.2.

\subsection{Target Density Fluctuations due to Zero-point Variations}

A further requirement of $e B O S S$ is that fluctuations in target density due to shifting zero-point calibrations across the SDSS imaging footprint are well controlled. Similar to Section 6.1, such fluctuations need to be kept below the $15 \%$ level (see also Section 2.2). ${ }^{55}$ To study how changes in zero-point affect the density of $e B O S S$ CORE quasar targets, each band used in the $e B O S S$ CORE quasar selection is offset by $\pm 0.01 \mathrm{mag}$ (i.e., scaled by $1 \%$ in flux) and the resulting fractional changes in target density are determined after re-running the target selection pipeline. Each SDSS band is tested individually. Because the WISE bands are only incorporated into eBOSS CORE quasar target selection in a stack (see Equation (2)),

\footnotetext{
55 This $15 \%$ limit is on the two-tailed distribution (i.e., between the peaks due to a positive and a negative fluctuation in zero-point).
} 

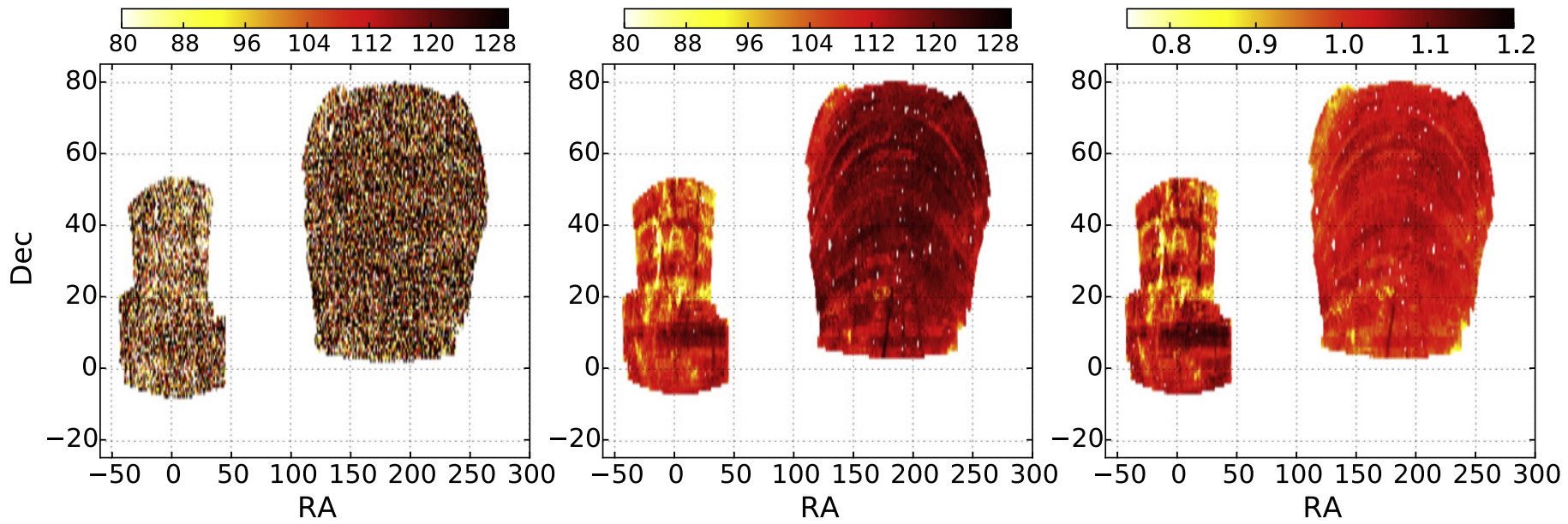

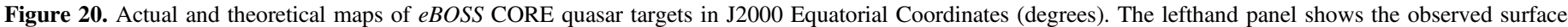

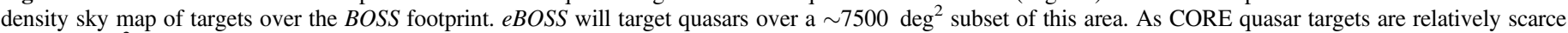

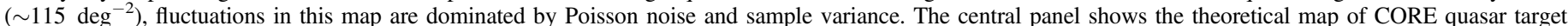

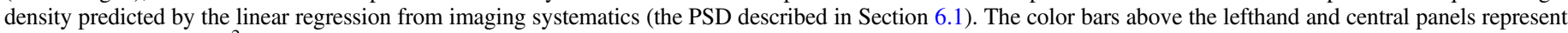

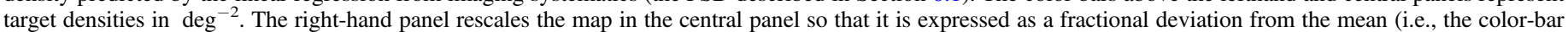
above this panel represents the quantity $\mathrm{PSD} /\langle\mathrm{PSD}\rangle$ ).

both $\mathrm{W} 1$ and $\mathrm{W} 2$ are simultaneously shifted by \pm 0.01 mag and the result is reported as a single band (henceforth denoted $W$ ).

The resulting fractional fluctuations in target density from these offsets $\left(N^{-1}[\Delta N / \Delta \mathrm{m}]\right)$ can then be multiplied by the zero-point rms error expected for the imaging calibrations used by eBOSS (see Section 3) to determine the expected rms variation in number density due to zero-point calibrations shifting across the $e B O S S$ footprint. We adopt the zero-point errors in $[u, g, r, i, z]$ of $[13,9,7,7,8]$ mmag rms from D. Finkbeiner et al. (2016, in preparation) and conservatively estimate a zero-point error of $20 \mathrm{mmag}$ rms for the $W$ stack (see Jarrett et al. 2011). Assuming that the zero-point errors can be modeled using a Gaussian distribution, 95\% of CORE quasar targets in $e B O S S$ will be within $\pm 2 \sigma$ of the expected rms variation. In other words, $95 \%$ fractional variance in target density can be interpreted as meaning that $95 \%$ of the area of the sky is expected to be described by fluctuations of $\pm 2 \sigma$. Thus, the overall $95 \%$ fractional variance in target density due to zero-point errors can be expressed (as a percentage) as $100 \% \times 4 \times$ [zero-point error $] \times\left[N^{-1}(\Delta N / \Delta \mathrm{m})\right]$. Table 6 displays the results of this analysis, which indicate that the $g$ band is the least robust to zero-point variations when selecting eBOSS CORE quasars. Even the $g$-band, however, causes a $(2 \sigma)$ variation of only $3 \%$, which is far less than the $15 \%$ limit outlined in Section 2.2. eBOSS CORE quasar target selection is thus completely robust to zero-point errors.

\section{CONCLUSIONS AND SUMMARY}

The fourth iteration of the SDSS will include $e B O S S$, a project with the overarching goal of using galaxies and quasars to measure the BAO scale across a range of redshifts. This paper details the construction of a sample of quasars that can provide the first $2 \%$ constraints on the BAO scale at redshifts $0.9<z<2.2$ through clustering measurements, referred to as the eBOSS "CORE" sample. The final eBOSS CORE algorithm, which is designed to be a homogeneous and reproducible selection, is as follows.

1. Take all targets in the D. Finkbeiner et al. (2016, in preparation) recalibrations of SDSS imaging, which are stored in the calib_obj or "Data Sweep" format (Blanton et al. 2005).

2. Select PRIMARY point sources (objc_type==6) that have (de-extincted) PSF magnitudes of $g<22$ or $r<22$, a FIBER2MAG of $i>17$, and good IMAGE_STATUS.

3. Apply the XDQSOz method of Bovy et al. (2012) to these sources and restrict to objects with PQSO $(z>0.9)>0.2$.

4. Force-photometer WISE imaging at the positions of the resulting sources using the Lang (2014) approach, or, equivalently, match to the force-photometered catalog of Lang et al. (2014).

5. Create band-weighted stacks from the fluxes of these sources using photometry from the SDSS $f_{\mathrm{opt}}=\left(f_{g}+\right.$ $\left.0.8 f_{r}+0.6 f_{i}\right) / 2.4$ and WISE $f_{W I S E}=\left(f_{W 1}+0.5 f_{W 2}\right) / 1.5$.

6. Convert these flux stacks to magnitudes and restrict to sources with $m_{\mathrm{opt}}-m_{W I S E} \geqslant(g-i)+3$.

The resulting set of sources comprise the eBOSS CORE quasar sample. Not all such sources, however, are targeted for spectroscopy in $e B O S S$. The eBOSS survey does not place a fiber on any target that has an existing good spectrum from earlier iterations of the SDSS (see Section 4.4.10).

This paper also describes a $z>2.1$ quasar sample that can be used to refine the BAO scale measured from clustering in the Ly $\alpha$ Forest, referred to as the eBOSS "Ly $\alpha$ " sample. The various techniques used to target $\operatorname{Ly} \alpha$ quasars for $e B O S S$ are not designed to be homogeneous and reproducible, so they are only discussed in full in the body of this paper (see, e.g., Figure 1).

The CORE and Ly $\alpha$ quasar targeting algorithms have been used to select targets for a spectroscopic survey over a large area in the SDSS NGC region, in order to test whether these algorithms meet the requirements for $e B O S S$. This $\sim 810 \mathrm{deg}^{2}$ survey is known as SEQUELS. Observations over the first $\sim 300 \mathrm{deg}^{2}$ of SEQUELS have been completed, and visual inspections of all SEQUELS targets are used to project outcomes for eBOSS (see, e.g., Table 4).

The algorithms developed in this paper meet all of the requirements of $e B O S S$ quasar targeting that can be projected 
NGC
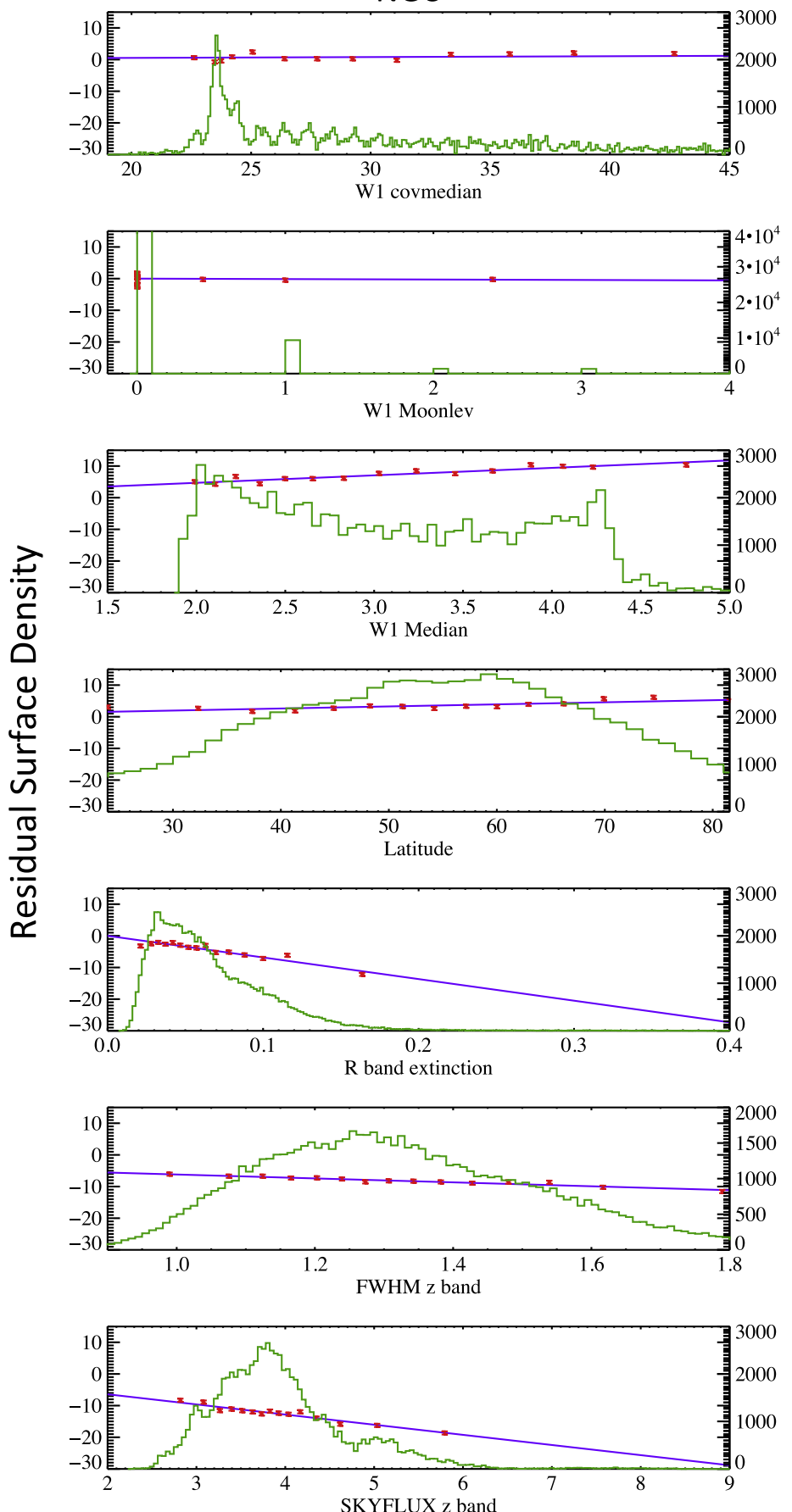

SGC
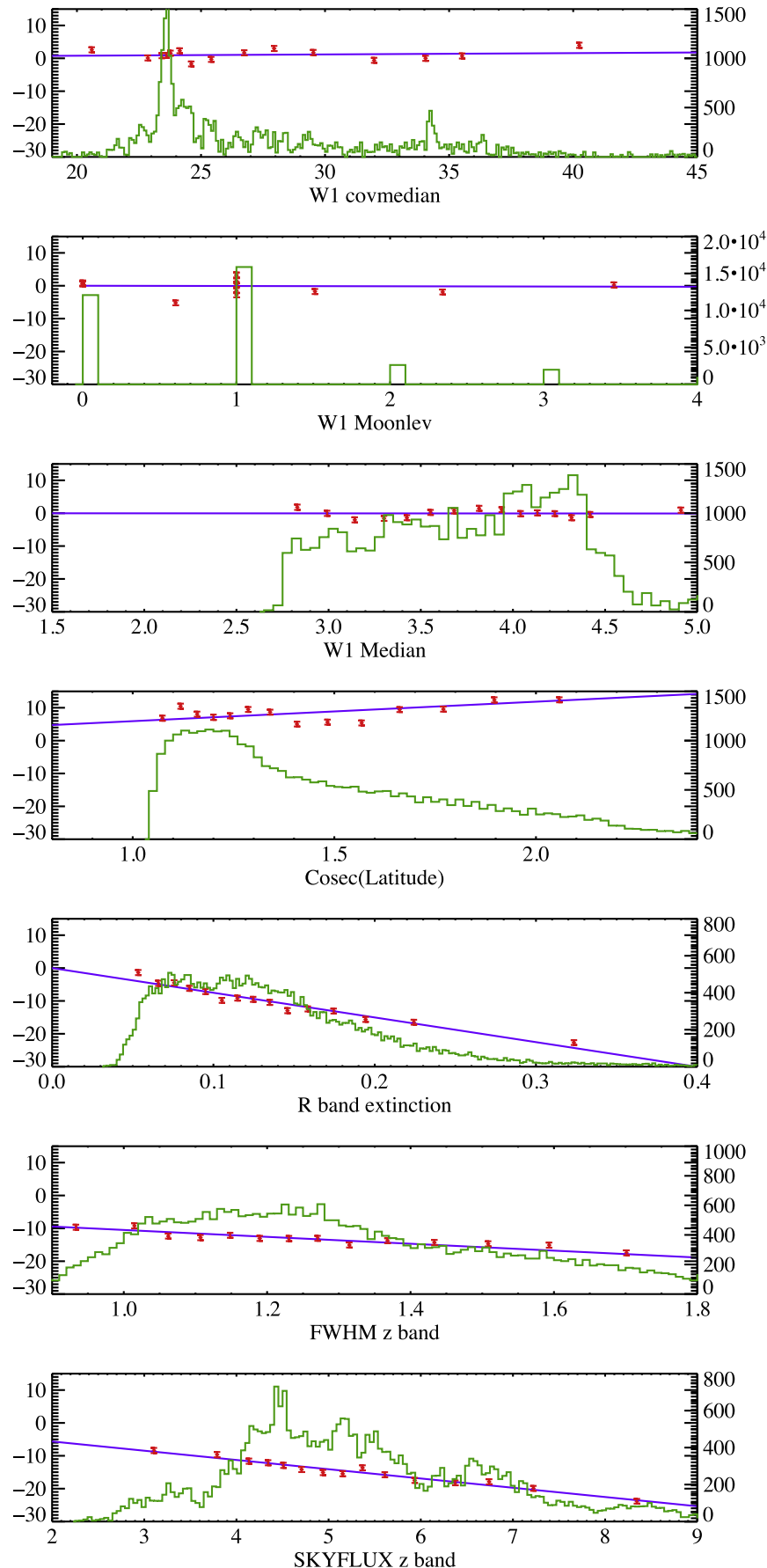

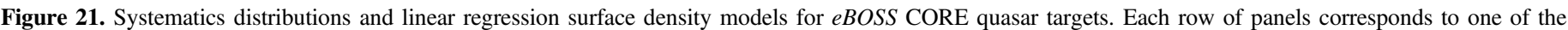

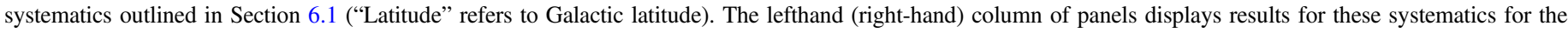

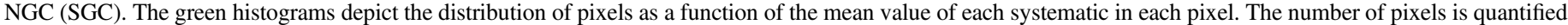

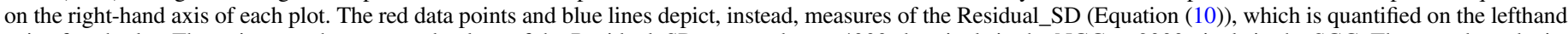

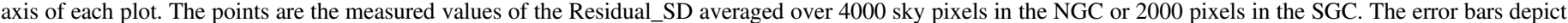

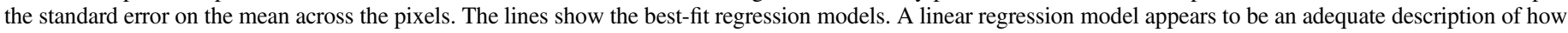
each displayed systematic affects $e B O S S$ CORE quasar target density.

from SEQUELS. In particular, the requisite number densities for $e B O S S$ are $>58 \mathrm{deg}^{-2}$ uniformly selected quasars in the redshift range $0.9<z<2.2$, leaving as many fibers as possible to target new Ly $\alpha$ quasars. Results from SEQUELS suggest that eBOSS will recover $\sim 70 \mathrm{deg}^{-2}$ $0.9<z<2.2$ quasars using the CORE selection technique and $\sim 10 \mathrm{deg}^{-2}$ new $z>2.1$ quasars from various Ly $\alpha$ selection techniques. ${ }^{56}$ In addition, the adopted SDSS and WISE imaging is sufficiently homogeneous for quasar targeting that the statistics projected from SEQUELS are expected to

\footnotetext{
56 These Ly $\alpha$ quasar densities will be reduced slightly by the fact that $P T F$ imaging is only expected to be available over $\sim 90 \%$ of the $e B O S S$ footprint, as detailed in our companion overview paper (Dawson et al. 2015).
} 
Table 6

Results of How Zero-point Fluctuations Affect Target Density

\begin{tabular}{lccc}
\hline \hline & $N^{-1}(\Delta N / \Delta \mathrm{m})$ & Zero-point Error & Fluctuation \\
& $(1)$ & $12)$ & $(3)$ \\
\hline$u$ & 0.544 & $9 \times 10^{-3}$ & $2.8 \%$ \\
$g$ & 0.856 & $7 \times 10^{-3}$ & $3.1 \%$ \\
$r$ & 0.514 & $7 \times 10^{-3}$ & $1.4 \%$ \\
$i$ & 0.475 & $8 \times 10^{-3}$ & $1.3 \%$ \\
$z$ & 0.061 & $20 \times 10^{-3}$ & $0.2 \%$ \\
$W$ & 0.223 & & $1.8 \%$ \\
\hline
\end{tabular}

Note. (1) Fractional deviation in target density that results from a $\pm 0.01 \mathrm{mag}$ scatter in each band; (2) Zero-point rms error in each band in magnitudes. Values for the SDSS are taken from D. Finkbeiner et al. (2016, in preparation). Values for the WISE stack are estimated from Jarrett et al. (2011); (3) 95\% $( \pm 2 \sigma)$ values in target density fluctuation corresponding to $100 \% \times 4 \times$ [zeropoint error $] \times\left[N^{-1}(\Delta N / \Delta \mathrm{m})\right]$.

remain valid over close to $90 \%$ of the $e B O S S$ footprint. The few eBOSS quasar sample requirements or assumptions that are not discussed in this paper are verified elsewhere. These include a survey area of at least $7500 \mathrm{deg}^{2}$ and precise and accurate redshifts for quasars (see Dawson et al. 2015).

Ultimately, eBOSS will uniformly target in excess of 500,000 quasars in the redshift range $0.9<z<2.2$, exceeding previous such clustering samples by a factor of more than 10 . Samples of new spectroscopically confirmed quasars across all redshifts in $e B O S S$ will exceed 500,000 quasars, which will be at least three times larger than all previous samples across the $e B O S S$ footprint in combination. At the conclusion of $e B O S S$, in excess of 800,000 confirmed quasars should have spectra from some iteration of the SDSS. In essence, $e B O S S$ will be the next-generation quasar survey, and, in the wake of 20 years of observations from SDSS-I, II, III, and IV, eBOSS will usher in the era of million-fold spectroscopic quasar samples.

We are grateful for insightful discussions about quasar selection statistics with Joe Hennawi, David Hogg, and Gordon Richards. A.D.M. acknowledges a generous research fellowship from the Alexander von Humboldt Foundation at the MaxPlanck-Institut für Astronomie and was supported in part by NASA-ADAP awards NNX12AI49G and NNX12AE38G and by NSF awards 1211112 and 1515404 . J.P.K. acknowledges support from the ERC advanced grant LIDA.

This paper includes targets derived from the images of the Wide-Field Infrared Survey Explorer, which is a joint project of the University of California, Los Angeles, and the Jet Propulsion Laboratory/California Institute of Technology, funded by the National Aeronautics and Space Administration.

This paper represents an effort by both the SDSS-III and SDSS-IV collaborations. Funding for SDSS-III was provided by the Alfred P. Sloan Foundation, the Participating Institutions, the National Science Foundation, and the U.S. Department of Energy Office of Science. Funding for the Sloan Digital Sky Survey IV has been provided by the Alfred P. Sloan Foundation, the U.S. Department of Energy Office of Science, and the Participating Institutions. SDSS-IV acknowledges support and resources from the Center for HighPerformance Computing at the University of Utah. The SDSS web site is www.sdss.org.

SDSS-IV is managed by the Astrophysical Research Consortium for the Participating Institutions of the SDSS
Collaboration, including the Brazilian Participation Group, the Carnegie Institution for Science, Carnegie Mellon University, the Chilean Participation Group, the French Participation Group, Harvard-Smithsonian Center for Astrophysics, Instituto de Astrofísica de Canarias, The Johns Hopkins University, Kavli Institute for the Physics and Mathematics of the universe (IPMU)/University of Tokyo, Lawrence Berkeley National Laboratory, Leibniz Institut für Astrophysik Potsdam (AIP), Max-Planck-Institut für Astronomie (MPIA Heidelberg), MaxPlanck-Institut für Astrophysik (MPA Garching), Max-PlanckInstitut für Extraterrestrische Physik (MPE), National Astronomical Observatory of China, New Mexico State University, New York University, University of Notre Dame, Observatário Nacional/MCTI, The Ohio State University, Pennsylvania State University, Shanghai Astronomical Observatory, United Kingdom Participation Group, Universidad Nacional Autónoma de México, University of Arizona, University of Colorado Boulder, University of Portsmouth, University of Utah, University of Virginia, University of Washington, University of Wisconsin, Vanderbilt University, and Yale University.

\section{REFERENCES}

Abazajian, K. N., Adelman-McCarthy, J. K., Agüeros, M. A., et al. 2009, ApJS, 182, 543

Aihara, H., Allende Prieto, C., An, D., et al. 2011, ApJS, 193, 29

Alam, S., Albareti, F. D., Allende Prieto, C., et al. 2015, ApJS, 219, 12 Alexandroff, R., Strauss, M. A., Greene, J. E., et al. 2013, MNRAS, 435, 3306 Assef, R. J., Stern, D., Kochanek, C. S., et al. 2013, ApJ, 772, 26

Aubourg, É., Bailey, S., Bautista, J. E., et al. 2014, arXiv:1411.1074

Banerji, M., McMahon, R. G., Hewett, P. C., et al. 2012, MNRAS, 427, 2275

Becker, R. H., White, R. L., \& Helfand, D. J. 1995, ApJ, 450, 559

Bertin, E. 2006, in ASP Conf. Ser. 351, Astronomical Data Analysis Software and Systems XV, ed. C. Gabriel et al. (San Francisco, CA: ASP), 112

Bertin, E., Mellier, Y., Radovich, M., et al. 2002, in ASP Conf. Ser. 281, Astronomical Data Analysis Software and Systems XI, ed. D. A. Bohlender, D. Durand \& T. H. Handley (San Francisco, CA: ASP), 228

Blain, A. W., Assef, R., Stern, D., et al. 2013, ApJ, 778, 113

Blanton, M. R., Lin, H., Lupton, R. H., et al. 2003, AJ, 125, 2276

Blanton, M. R., Schlegel, D. J., Strauss, M. A., et al. 2005, AJ, 129, 2562

Bolton, A. S., Schlegel, D. J., Aubourg, É., et al. 2012, AJ, 144, 144 Boroson, T. A., \& Lauer, T. R. 2009, Natur, 458, 53

Bovy, J., Hennawi, J. F., Hogg, D. W., et al. 2011a, ApJ, 729, 141

Bovy, J., Hogg, D. W., \& Roweis, S. T. 2011b, AnApS, 5, 1657

Bovy, J., Myers, A. D., Hennawi, J. F., et al. 2012, ApJ, 749, 41

Boyle, B. J., Fong, R., Shanks, T., \& Peterson, B. A. 1990, MNRAS, 243, 1

Braccesi, A., Formiggini, L., \& Gandolfi, E. 1970, A\&A, 5, 264

Busca, N. G., Delubac, T., Rich, J., et al. 2013, A\&A, 552, A96

Butler, N. R., \& Bloom, J. S. 2011, AJ, 141, 93

Cimatti, A., Zamorani, G., \& Marano, B. 1993, MNRAS, 263, 236

Claeskens, J.-F., Smette, A., Vandenbulcke, L., \& Surdej, J. 2006, MNRAS, 367,879

Comparat, J., Delubac, T., Jouvel, S., et al. 2015, arXiv:1509.05045

Cristiani, S., Trentini, S., La Franca, F., et al. 1996, A\&A, 306, 395

Croom, S. M., Richards, G. T., Shanks, T., et al. 2009, MNRAS, 392, 19

Croom, S. M., Smith, R. J., Boyle, B. J., et al. 2004, MNRAS, 349, 1397

Dawson, K. S., Kneib, J.-P., Percival, W. J., et al. 2015, arXiv:1508.04473

Dawson, K. S., Schlegel, D. J., Ahn, C. P., et al. 2013, AJ, 145, 10

Delubac, T., Bautista, J. E., Busca, N. G., et al. 2015, A\&A, 574, A59

Eftekharzadeh, S., Myers, A. D., White, M., et al. 2015, MNRAS, 453, 2779

Eisenstein, D. J., Hu, W., \& Tegmark, M. 1998, ApJL, 504, L57

Eisenstein, D. J., Weinberg, D. H., Agol, E., et al. 2011, AJ, 142, 72

Fan, X. 1999, AJ, 117, 2528

Filiz, Ak, N., Brandt, W. N., Hall, P. B., et al. 2012, ApJ, 757, 114

Filiz, Ak, N., Brandt, W. N., Hall, P. B., et al. 2013, ApJ, 777, 168

Filiz, Ak, N., Brandt, W. N., Hall, P. B., et al. 2014, ApJ, 791, 88

Finley, H., Petitjean, P., Pâris, I., et al. 2013, A\&A, 558, A111

Fitzpatrick, E. L. 1999, PASP, 111, 63

Font-Ribera, A., Kirkby, D., Busca, N., et al. 2014, JCAP, 5, 27

Formiggini, L., Zitelli, V., Bonoli, F., \& Braccesi, A. 1980, A\&AS, 39, 129

Fukugita, M., Ichikawa, T., Gunn, J. E., et al. 1996, AJ, 111, 1748 
Giveon, U., Maoz, D., Kaspi, S., Netzer, H., \& Smith, P. S. 1999, MNRAS, 306,637

Glikman, E., Urrutia, T., Lacy, M., et al. 2012, ApJ, 757, 51

Green, R. F., Schmidt, M., \& Liebert, J. 1986, ApJS, 61, 305

Greene, J. E., Alexandroff, R., Strauss, M. A., et al. 2014, ApJ, 788, 91

Gunn, J. E., Carr, M., Rockosi, C., et al. 1998, AJ, 116, 3040

Gunn, J. E., Siegmund, W. A., Mannery, E. J., et al. 2006, AJ, 131, 2332

Hainline, K. N., Hickox, R. C., Carroll, C. M., et al. 2014, ApJ, 795, 124

Hawkins, M. R. S. 1983, MNRAS, 202, 571

Helfand, D. J., White, R. L., \& Becker, R. H. 2015, ApJ, 801, 26

Hennawi, J. F., Strauss, M. A., Oguri, M., et al. 2006, AJ, 131, 1

Hewett, P. C., Foltz, C. B., \& Chaffee, F. H. 1995, AJ, 109, 1498

Hewett, P. C., \& Wild, V. 2010, MNRAS, 405, 2302

Hickox, R. C., Jones, C., Forman, W. R., et al. 2007, ApJ, 671, 1365

Hickox, R. C., Jones, C., Forman, W. R., et al. 2009, ApJ, 696, 891

Inada, N., Oguri, M., Pindor, B., et al. 2003, Natur, 426, 810

Jarrett, T. H., Cohen, M., Masci, F., et al. 2011, ApJ, 735, 112

Kaiser, N., Burgett, W., Chambers, K., et al. 2010, Proc. SPIE, 7733, 7330E

Kaspi, S., Brandt, W. N., Maoz, D., et al. 2007, ApJ, 659, 997

Kennefick, J. D., de Carvalho, R. R., Djorgovski, S. G., et al. 1995, AJ, 110, 78

Kirkby, D., Margala, D., Slosar, A., et al. 2013, JCAP, 3, 24

Kozłowski, S., Kochanek, C. S., Udalski, A., et al. 2010, ApJ, 708, 927

Kron, R. G., \& Chiu, L.-T. G. 1981, PASP, 93, 397

Lacy, M., Petric, A. O., Sajina, A., et al. 2007, AJ, 133, 186

Lacy, M., Ridgway, S. E., Gates, E. L., et al. 2013, ApJS, 208, 24

Lacy, M., Storrie-Lombardi, L. J., Sajina, A., et al. 2004, ApJS, 154, 166

Laher, R. R., Surace, J., Grillmair, C. J., et al. 2014, PASP, 126, 674

Lang, D. 2014, AJ, 147, 108

Lang, D., Hogg, D. W., \& Schlegel, D. J. 2014, arXiv:1410.7397

Law, N. M., Kulkarni, S. R., Dekany, R. G., et al. 2009, PASP, 121, 1395

Linder, E. V. 2003, PhRvD, 68, 083504

Lupton, R. H., Gunn, J. E., \& Szalay, A. S. 1999, AJ, 118, 1406

MacLeod, C. L., Brooks, K., Ivezić, Ž., et al. 2011, ApJ, 728, 26

MacLeod, C. L., Ivezić, Ž., Kochanek, C. S., et al. 2010, ApJ, 721, 1014

McGreer, I. D., Helfand, D. J., \& White, R. L. 2009, AJ, 138, 1925

McGreer, I. D., Jiang, L., Fan, X., et al. 2013, ApJ, 768, 105

McLure, R. J., \& Dunlop, J. S. 2004, MNRAS, 352, 1390

Morganson, E., Green, P. J., Anderson, S. F., et al. 2015, ApJ, 806, 244

Netzer, H., \& Trakhtenbrot, B. 2007, ApJ, 654, 754

Newberg, H. J., \& Yanny, B. 1997, ApJS, 113, 89

Noterdaeme, P., Petitjean, P., Carithers, W. C., et al. 2012, A\&A, 547, L1

Ofek, E. O., Laher, R., Law, N., et al. 2012, PASP, 124, 62

Oke, J. B., \& Gunn, J. E. 1983, ApJ, 266, 713

Osmer, P. S. 1982, ApJ, 253, 28

Padmanabhan, N., Schlegel, D. J., Finkbeiner, D. P., et al. 2008, ApJ, 674, 1217

Palanque-Delabrouille, N., Magneville, C., Yèche, C., et al. 2013a, A\&A, 551, A29

Palanque-Delabrouille, N., Magneville, C., Yèche, C., et al. 2015, arXiv: 1509.05607

Palanque-Delabrouille, N., Yèche, C., Borde, A., et al. 2013b, A\&A, 559, A85

Palanque-Delabrouille, N., Yeche, C., Myers, A. D., et al. 2011, A\&A, 530, A122
Pâris, I., Petitjean, P., Aubourg, É., et al. 2014, A\&A, 563, A54

Planck Collaboration, Ade, P. A. R., Aghanim, N., et al. 2014, A\&A, 571, A16 Prakash, A., Licquia, T. C., Newman, J. A., \& Rao, S. M. 2015a, ApJ, 803, 105 Prakash, A., Licquia, T. C., Newman, J. A., et al. 2015b, arXiv:1508.04478 Rau, A., Kulkarni, S. R., Law, N. M., et al. 2009, PASP, 121, 1334 Rengstorf, A. W., Brunner, R. J., \& Wilhite, B. C. 2006, AJ, 131, 1923 Rengstorf, A. W., Mufson, S. L., Abad, C., et al. 2004a, ApJ, 606, 741 Rengstorf, A. W., Mufson, S. L., Andrews, P., et al. 2004b, ApJ, 617, 184 Richards, G. T., Deo, R. P., Lacy, M., et al. 2009a, AJ, 137, 3884 Richards, G. T., Fan, X., Newberg, H. J., et al. 2002, AJ, 123, 2945 Richards, G. T., Fan, X., Schneider, D. P., et al. 2001, AJ, 121, 2308 Richards, G. T., Myers, A. D., Gray, A. G., et al. 2009b, ApJS, 180, 67 Richards, G. T., Strauss, M. A., Fan, X., et al. 2006, AJ, 131, 2766 Ross, A. J., Ho, S., Cuesta, A. J., et al. 2011, MNRAS, 417, 1350 Ross, N. P., McGreer, I. D., White, M., et al. 2013, ApJ, 773, 14 Ross, N. P., Myers, A. D., Sheldon, E. S., et al. 2012, ApJS, 199, 3 Sandage, A. 1965, ApJ, 141, 1560

Sandage, A., \& Luyten, W. J. 1969, ApJ, 155, 913

Schlafly, E. F., \& Finkbeiner, D. P. 2011, ApJ, 737, 103

Schlafly, E. F., Finkbeiner, D. P., Jurić, M., et al. 2012, ApJ, 756, 158

Schlegel, D. J., Finkbeiner, D. P., \& Davis, M. 1998, ApJ, 500, 525

Schmidt, K. B., Marshall, P. J., Rix, H.-W., et al. 2010, ApJ, 714, 1194

Schmidt, M. 1963, Natur, 197, 1040

Schmidt, M., Schneider, D. P., \& Gunn, J. E. 1986, ApJ, 306, 411

Schneider, D. P., Richards, G. T., Hall, P. B., et al. 2010, AJ, 139, 2360

Scranton, R., Johnston, D., Dodelson, S., et al. 2002, ApJ, 579, 48

Seo, H.-J., \& Eisenstein, D. J. 2003, ApJ, 598, 720

Sesar, B., Ivezić, Ž., Lupton, R. H., et al. 2007, AJ, 134, 2236

Shen, Y., Greene, J. E., Strauss, M. A., Richards, G. T., \& Schneider, D. P. 2008, ApJ, 680, 169

Slosar, A., Font-Ribera, A., Pieri, M. M., et al. 2011, JCAP, 9, 1

Slosar, A., Iršič, V., Kirkby, D., et al. 2013, JCAP, 4, 26

Smee, S. A., Gunn, J. E., Uomoto, A., et al. 2013, AJ, 146, 32

Stern, D., Assef, R. J., Benford, D. J., et al. 2012, ApJ, 753, 30

Stern, D., Eisenhardt, P., Gorjian, V., et al. 2005, ApJ, 631, 163

Stoughton, C., Lupton, R. H., Bernardi, M., et al. 2002, AJ, 123, 485

Swanson, M. E. C., Tegmark, M., Hamilton, A. J. S., \& Hill, J. C. 2008, MNRAS, 387, 1391

Trichas, M., Green, P. J., Silverman, J. D., et al. 2012, ApJS, 200, 17

Usher, P. D. 1978, ApJ, 222, 40

van den Bergh, S., Herbst, E., \& Pritchet, C. 1973, AJ, 78, 375

Vanden Berk, D. E., Richards, G. T., Bauer, A., et al. 2001, AJ, 122, 549

Vanden Berk, D. E., Wilhite, B. C., Kron, R. G., et al. 2004, ApJ, 601, 692

Vikas, S., Wood-Vasey, W. M., Lundgren, B., et al. 2013, ApJ, 768, 38

Voges, W., Aschenbach, B., Boller, T., et al. 1999, A\&A, 349, 389

Warren, S. J., Hewett, P. C., Irwin, M. J., McMahon, R. G., \& Bridgeland, M. T. 1987, Natur, 325, 131

White, M., Myers, A. D., Ross, N. P., et al. 2012, MNRAS, 424, 933

White, R. L., Becker, R. H., Gregg, M. D., et al. 2000, ApJS, 126, 133

Wright, E. L., Eisenhardt, P. R. M., Mainzer, A. K., et al. 2010, AJ, 140, 1868

Yan, L., Donoso, E., Tsai, C.-W., et al. 2013, AJ, 145, 55

York, D. G., Adelman, J., Anderson, J. E., Jr., et al. 2000, AJ, 120, 1579

York, D. G., Khare, P., Vanden Berk, D., et al. 2006, MNRAS, 367, 945 